\title{
Nearby early-type galaxies with ionized gas
}

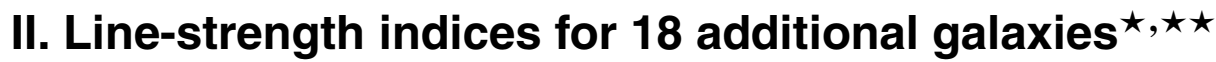

\author{
F. Annibali ${ }^{1}$, A. Bressan ${ }^{1,2}$, R. Rampazzo ${ }^{2}$, and W. W. Zeilinger ${ }^{3}$ \\ 1 SISSA, via Beirut 4, 34014 Trieste, Italy \\ e-mail: annibali@sissa.it, annibali@stsci.edu \\ 2 INAF - Osservatorio Astronomico di Padova, vicolo dell'Osservatorio 5, 35122 Padova, Italy \\ 3 Institut für Astronomie der Universität Wien, Türkenschanzstraße 17, 1180 Wien, Austria
}

Received 4 July 2005 / Accepted 4 August 2005

\section{ABSTRACT}

We previously presented a data-set of line-strength indices for 50 early-type galaxies in the nearby Universe. The galaxy sample is biased toward galaxies showing emission lines, located in environments corresponding to a broad range of local galaxy densities, although predominantly in low density environments. The present addendum enlarges the above data-set of line-strength indices by analyzing 18 additional early-type galaxies (three galaxies, NGC 3607, NGC 5077 and NGC 5898 were presented in the previous set). We measured 25 line-strength indices, defined by the Lick IDS "standard" system (Trager et al. 1998, ApJS, 116, 1; Worthey \& Ottaviani 1997, ApJS, 111, 377), for 7 luminosity weighted apertures and 4 gradients of each galaxy. This addendum presents the line-strength data-set and compares it with the available data in the literature.

Key words. galaxies: elliptical and lenticular, $\mathrm{cD}$ - galaxies: fundamental parameters - galaxies: formation - galaxies: evolution

\section{Introduction}

The aim of our study is to improve our understanding of the nature of the ionized gas in early-type galaxies by studying its physical conditions, the possible ionization mechanisms, relations with the other gas components of the Inter Stellar Medium (ISM) and the connection with the stellar population of the host galaxy. By investigating issues such as the evolution of stellar populations and the ISM, we will both explore the complex, evolving ecosystem within early-type galaxies and build a database of well studied galaxies to be used as a reference set for the study of intermediate and distant objects. Our target is to characterize the stellar populations, with particular concern for those in the extended emission regions, through the modelling of the complete (lines and continuum) spectrum characteristics. This will allow us to constrain the galaxy formation/evolution history.

The adopted strategy, described in Rampazzo et al. (2005) (hereafter Paper I), consists of investigating the galaxy underlying stellar populations and the emission line properties at different galactocentric distances. The study of stellar populations in early-type galaxies is of fundamental importance

\footnotetext{
* Based on observations obtained at the European Southern Observatory, La Silla, Chile (Programs Nr. 60.A-0647 and 61.A-0406.

$\star \star$ Tables 5, 6, 8, 9 and Appendix A are only available in electronic form at http://www. edpsciences.org
}

to the understanding of their evolution with time (see e.g. Buzzoni et al. 1992; Worthey 1992; González 1993; Buzzoni et al. 1994; Worthey et al. 1994; Leonardi \& Rose 1996; Worthey \& Ottaviani 1997; Trager et al. 1998; Longhetti et al. 1998a; Vazdekis 1999; Longhetti et al. 1999; Longhetti et al. 2000; Trager et al. 2000; Kuntschner 2000; Beuing et al. 2002; Kuntschner et al. 2002; Thomas et al. 2003; Mehlert et al. 2003).

This addendum intends to enlarge the sample of the earlytype galaxies analyzed in Paper I providing the data-set of line-strength indices for 18 objects. Three galaxies, namely NGC 3607, NGC 5077 and NGC 5898 were already presented in the previous set and are used in this paper in order to check the internal consistency of our results on repeated observations. Eight galaxies in the present sample, NGC 3818, NGC 4374, NGC 4697, NGC 5044, NGC 5638, NGC 5812, NGC 5813 and NGC 5831, belong to the Trager et al. (1998) sample, six of which belong to the original González (1993) sample.

This note is organized as follows. Section 2 presents the characteristics of the sample of the 18 galaxies. Sections 3 and 4 summarize the observations, the reduction procedure, the line-strength index measurements at different galactocentric distances and the corrections applied to conform the indices to the Lick IDS system. In Sect. 5 we present the results and a comparison with the literature. 
Table 1. Overview of the observed sample.

\begin{tabular}{|c|c|c|c|c|c|c|c|c|c|c|c|c|}
\hline Ident & RA $(200$ & 0) Dec & RSA & $\mathrm{RC} 3$ & PA & $B_{0}$ & $(B-V)_{0}$ & $(U-B)_{0}$ & $V_{\text {hel }}$ & $r_{\mathrm{e}}$ & $\rho_{x y z}$ & $\epsilon$ \\
\hline NGC 3607 & 111654.3 & 180310 & S03(3) & $\mathrm{SA}(\mathrm{s}) 0:$ & 120 & 11.08 & 0.88 & 0.43 & 934 & 43.4 & 0.34 & 0.11 \\
\hline NGC 3818 & 114157.3 & -060920 & E5 & E5 & 103 & 12.47 & 0.91 & & 1701 & 22.2 & 0.20 & 0.36 \\
\hline NGC 4374 & 122503.7 & 125313 & E1 & E1 & 135 & 10.01 & 0.94 & 0.50 & 1060 & 50.9 & 3.99 & 0.11 \\
\hline NGC 4696 & 124849.3 & -411840 & (E3) & $\mathrm{E}+1 \mathrm{pec}$ & 95 & 10.85 & & & 2958 & 85.0 & 0.00 & 0.34 \\
\hline NGC 4697 & 124835.9 & -054803 & E6 & E6 & 70 & 10.07 & 0.89 & 0.39 & 1241 & 72.0 & 0.60 & 0.32 \\
\hline NGC 5011 & 131251.8 & -430546 & E2 & E1-2 & 154 & 11.90 & 0.89 & & 3104 & 23.8 & 0.27 & 0.15 \\
\hline NGC 5044 & 131524.0 & -162308 & E0 & E0 & 11 & 11.67 & & & 2704 & 82.3 & 0.38 & 0.11 \\
\hline NGC 5077 & 131931.6 & -123926 & S01/2(4) & E3+ & 11 & 12.52 & 0.98 & 0.54 & 2764 & 22.8 & 0.23 & 0.15 \\
\hline NGC 5090 & 132112.8 & -434216 & E2 & E2 & 136 & 11.97 & & & 3421 & 62.4 & & 0.15 \\
\hline NGC 5193 & 133153.5 & -331404 & S01(0) & E pec & 71 & 12.35 & 0.86 & & 3711 & 26.7 & & 0.07 \\
\hline NGC 5266 & 134302.1 & -481010 & S03(5) pec & SA0-: & 109 & 11.50 & & & 3074 & 76.7 & 0.35 & 0.31 \\
\hline NGC 5638 & 142940.4 & 031400 & E1 & E1 & 150 & 12.06 & 0.91 & 0.46 & 1676 & 28.0 & 0.79 & 0.11 \\
\hline NGC 5812 & 150055.7 & -072726 & E0 & E0 & 130 & 11.83 & 0.94 & & 1930 & 25.5 & 0.19 & 0.13 \\
\hline NGC 5813 & 150111.2 & 014207 & E1 & E1-2 & 145 & 11.42 & 0.94 & 0.52 & 1972 & 57.2 & 0.88 & 0.28 \\
\hline NGC 5831 & 150407.0 & 011312 & E4 & E3 & 55 & 12.31 & 0.92 & 0.55 & 1656 & 25.5 & 0.83 & 0.13 \\
\hline NGC 5898 & 151813.6 & -240551 & S02/3(0) & E0 & 30 & 12.41 & 0.95 & & 2267 & 22.2 & 0.23 & 0.07 \\
\hline NGC 6758 & 191352.3 & -561836 & E2 (merger) & $E+:$ & 121 & 12.31 & 0.96 & 0.44 & 3404 & 20.3 & & 0.22 \\
\hline NGC 6776 & 192519.1 & -635137 & E1 pec & $\mathrm{E}+$ pec & 15 & 12.71 & 0.86 & 0.44 & 5480 & 17.7 & & 0.17 \\
\hline
\end{tabular}

Notes: the value of $r_{\mathrm{e}}$ of NGC 4696, NGC 5090 and NGC 5266 have been derived from ESO-LV (Lauberts \& Valentijn 1989) while the value for NGC 5044 is derived from Goudfrooij (1994).

\section{Characterization of the sample}

Like the original sample of 50 early-type galaxies presented in Paper I, this sample of 18 galaxies is selected from a compilation of galaxies showing ISM traces in at least one of the following bands: IRAS $100 \mu \mathrm{m}, \mathrm{X}$-ray, radio, HI and CO (Roberts et al. 1991). All galaxies belong to the Revised Shapley Ames Catalog of Bright Galaxies (RSA) (Sandage \& Tammann 1987) and have a redshift of less than $5500 \mathrm{~km} \mathrm{~s}^{-1}$. The sample should then be biased towards objects that might be expected to have ongoing and recent star formation, at least in small amounts, because of the presence of emission lines. The emission should come from a combination of active galactic nuclei and star formation regions within the galaxies. Table 1 summarizes the basic characteristics of the galaxies available from the literature. Column 1 provides the identification; Cols. 2 and 3 the RA and Dec coordinates; Cols. 4 and 5 the galaxy morphological classifications according to the RSA (Sandage \& Tamman 1987) and RC3 (de Vaucouleurs et al. 1991) respectively. Columns 6-9 give the position angle of the isophotes along the major axis, the total corrected magnitude and the total $(B-V)$ and $(U-B)$ corrected colors from RC3 respectively. The heliocentric systemic velocity from HYPERCAT (http://www-obs.univ-lyon1.fr/hypercat) is reported in Col. 10. The effective radius, derived from $A_{\mathrm{e}}$, the diameter of the effective aperture from RC3, is given in Col. 11. A measure of the richness of the environment, $\rho_{x y z}\left(\right.$ galaxies $\mathrm{Mpc}^{-3}$ ), surrounding each galaxy is reported in Col. 12 (Tully 1988). Column 13 lists the average ellipticity of the galaxy as obtained from HYPERCAT.
Figure 1 summarizes the basic characteristics of the total sample $(50+15$ new galaxies of this paper $)$ and in particular, in the right bottom panel, provides evidence that a large fraction of galaxies is in low density environments.

Excluding NGC 3607, NGC 5077 and NGC 5898 already discussed in Paper I, seven of the new galaxies in the present sample belong to the sample of 73 luminous early-type galaxies selected from the RC3 catalogue by Macchetto et al. (1996) for a characterization of the extended emission region in $\mathrm{H} \alpha+[\mathrm{NII}]$ emission lines. The seven galaxies are NGC 5044, NGC 5090, NGC 5812, BGC 5813, NGC 5831, NGC 6758 and NGC 6776. Macchetto et al. (1996) showed that in three of these galaxies, namely NGC 5044, NGC 5813 and NGC 6776, the shape of the emission region is filamentary $(\mathrm{F})$. In the remaining galaxies the ionized gas morphology is more regular, (NGC 6758), or reminiscent of a small disk (SD), (NGC 5090, NGC 5812 and NGC 5831).

In Appendix A we have collected individual notes on galaxies, emphasizing kinematic studies of the ionized gas component, its correlation with the stellar body and its possible origin. Information for NGC 3607, NGC 5077 and NGC 5898 are given in Paper I.

Several classes of galaxies are present in this additional sample: interacting or post-interacting galaxies, galaxies showing evidence of kinematic decoupling between galaxy subcomponents, elliptical galaxies with a dust lane along the minor axis, radio galaxies and galaxies hosting an AGN nucleus. To summarize the individual notes in Appendix A, the sample contains one galaxy showing a shell structure, namely NGC 6776, and a galaxy, NGC 5266, considered an "old merger" of 

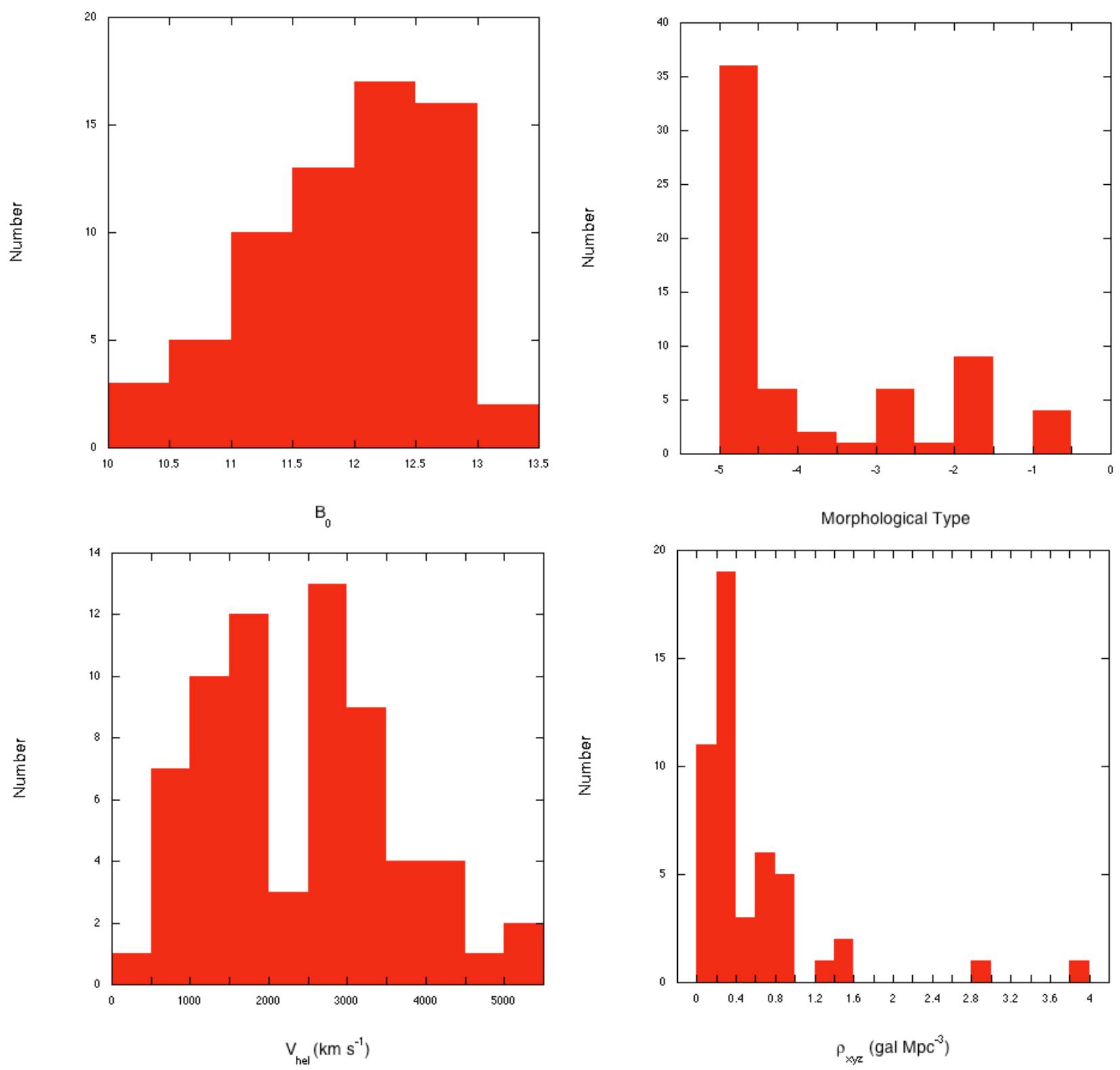

Fig. 1. Distribution of B-magnitudes (top left panel), morphological types (top right panel), heliocentric velocity (bottom left panel) and galaxy density (bottom right panel) for the total sample (50 galaxies of Paper I + 15 new galaxies of this paper).

two spirals having a large HI content. Six galaxies (namely NGC 4697, NGC 5077, NGC 5266, NGC 5813, NGC 5898 and NGC 6758) have a peculiar kinematic behaviour, e.g. counterrotation of stars vs. gas and/or stars vs. stars. Three galaxies (namely NGC 4374, NGC 4696 and NGC 5090) are FRI type radio sources. Often the ionized gas is associated to dustlane complexes (e.g. NGC 4374, NGC 4696, NGC 5266 and NGC 5813, see e.g. Goudfrooij 1994; Goudfrooij 1998). Some objects, as NGC 5638 and NGC 5813, seem finally "unperturbed" elliptical.

\section{Observations and data reduction}

\subsection{Observations}

Galaxies were observed between May 10 and 13th, 1999 at the $1.5 \mathrm{~m}$ ESO telescope (La Silla), equipped with a
Boller \& Chivens spectrograph and a UV coated CCD Fa2048L $(2048 \times 2048)$ camera (ESO CCD \#39). Details of the observations and typical seeing during the run are reported in Table 2. Table 3 provides a journal of observations, i.e. the object identification (Col. 1), the slit position angle oriented North through East (Col. 2) and the total exposure time (Col. 3) and observing conditions (Col. 4). The spectroscopic slit was oriented along the galaxy major axis for most observations. HeAr-FeNe calibration frames were taken before and after each exposure to allow an accurate wavelength calibration.

\subsection{Data reduction, extraction of apertures and gradients}

We adopted the same data reduction procedure described in Paper I. We recall here that the fringing seriously affected 

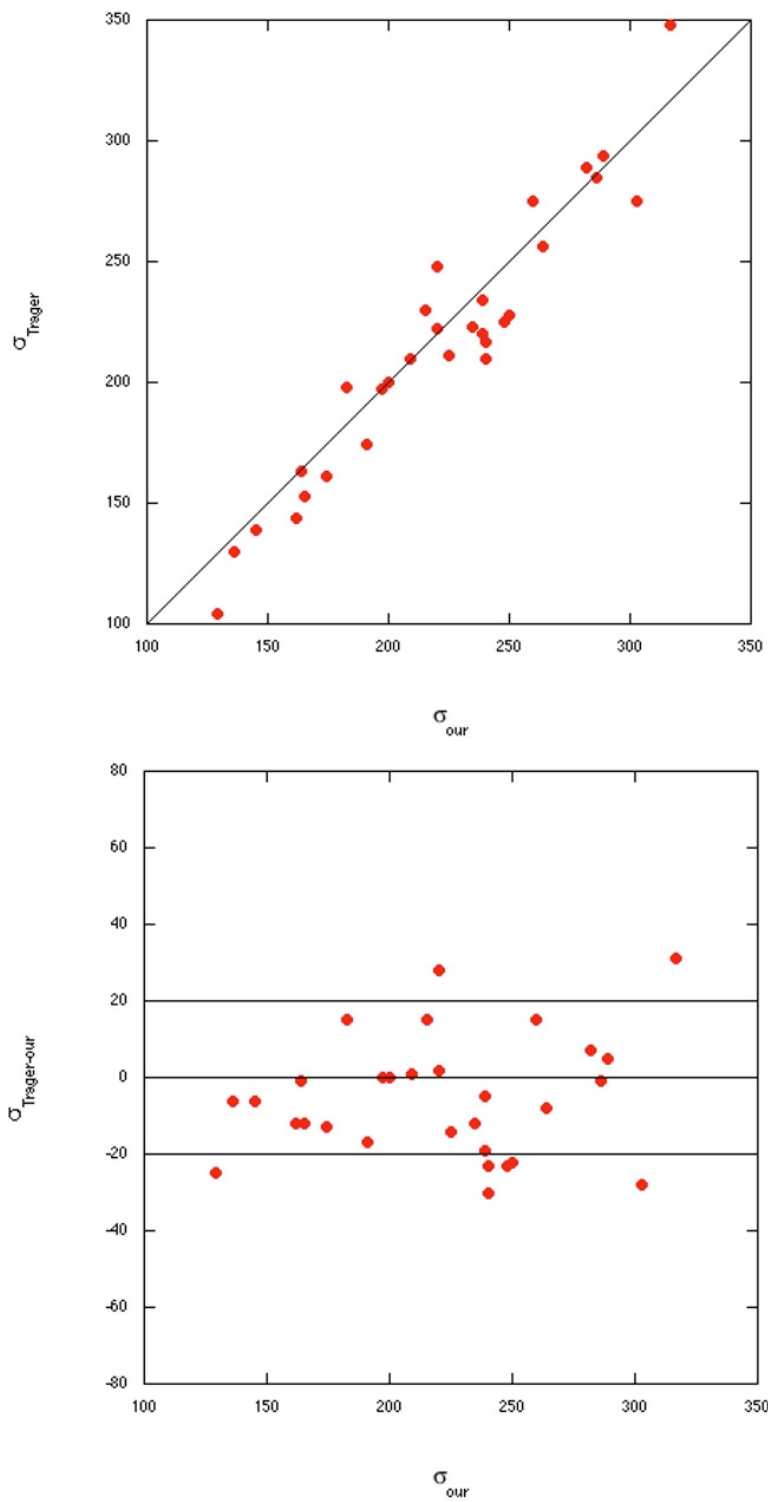

Fig. 2. Comparison between central velocity dispersions used in this paper and in Trager et al. (1998). The lines indicate an average error $\left(20 \mathrm{~km} \mathrm{~s}^{-1}\right)$ in the central velocity dispersion measurements.

observations longward of $\approx 7300 \AA$. After accurate flat-fielding correction, we considered the wavelength range 3700-7250 for further use. Multiple spectra for a given galaxy were coadded and flux calibrated using a sequence of spectrophotometric standard stars.

The definition of the apertures and gradients and their extraction procedure are detailed in Paper I. Summarizing, we have extracted flux-calibrated spectra along the slit in seven circular concentric regions, hereafter "apertures", and in four adjacent regions, hereafter "gradients". The seven luminosity weighted apertures, corrected for the galaxy ellipticity, have radii of $1.5^{\prime \prime}, 2.5^{\prime \prime}, 10^{\prime \prime}, r_{\mathrm{e}} / 10, r_{\mathrm{e}} / 8, r_{\mathrm{e}} / 4$ and $r_{\mathrm{e}} / 2$. The four gradients are derived in the regions $0 \leq r \leq r_{\mathrm{e}} / 16, r_{\mathrm{e}} / 16 \leq r \leq r_{\mathrm{e}} / 8$, $r_{\mathrm{e}} / 8 \leq r \leq r_{\mathrm{e}} / 4$ and $r_{\mathrm{e}} / 4 \leq r \leq r_{\mathrm{e}} / 2$.

\section{Measurements of line-strength indices and transformation into the Lick IDS system}

The procedure adopted to extract line-strength indices in the Lick IDS system from the galaxy spectra has been widely described in Paper I. Here we summarize the basic steps.

Since our spectral resolution $(F W H M \sim 7.6 \AA$ at $\sim 5000 \AA)$ on the entire spectrum is slightly better than the wavelengthdependent resolution of the Lick IDS system (see Worthey \& Ottaviani 1997), we have degraded our data convolving each spectrum (apertures and gradients) with a wavelengthdependent Gaussian kernel (see Eq. (5) of Paper I). On the smoothed spectra we have measured 25 line-strength indices: 21 of the original Lick IDS system (see Table 2 in Trager et al. 1998 for the index bandpass definitions) plus 4 higher order Balmer lines later introduced by Worthey \& Ottaviani (1997) (see their Table 1 for the index definitions).

\subsection{Correction for velocity dispersion}

The observed spectrum of a galaxy can be regarded as a stellar spectrum convolved with the radial velocity distribution of its stellar population. Therefore spectral features in a galactic spectrum are not the simple sum of its corresponding stellar spectra, because of the stellar motions. To measure the stellar composition of galaxies, we need to correct index measurements for the effects of the galaxy velocity dispersion (see e.g. González 1993 (hereafter G93), Trager et al. 1998; Longhetti et al. 1998a).

To this purpose in Paper I we selected stars with spectral type between G8III and K2III among the Lick stars observed together with the galaxies, and convolved their spectra with Gaussian curves of various widths in order to simulate different galactic velocity dispersions. On each convolved spectrum we then measured 25 Lick-indices and derived the fractional index variations as function of the velocity dispersion $\sigma$ :

$R_{i, j, \sigma}=\left(\frac{E W_{i, j, \sigma}-E W_{i, j, 0}}{E W_{i, j, 0}}\right)$

where $R_{i, j, \sigma}$ is the fractional variation of the $i$ th index measured for the $j$ th star at the velocity dispersion $\sigma$, while $E W_{i, j, \sigma}$ and $E W_{i, j, 0}$ are the index values at the velocity dispersion $\sigma$ and at zero velocity dispersion respectively. To correct the galactic indices we used a mean correction derived through an average on the $R_{i, j, \sigma}$ values (see Sect. 4.2 of Paper I for details).

The use of an average correction is a reasonable choice as long as the fractional index change $R_{i, j, \sigma}$ does not depend on the index value at fixed velocity dispersion. On the contrary we have verified that in some cases the fractional index change presents a correlation with the line strength index, as shown in Fig. 3 for Mgb, and the use of an average velocity dispersion correction may be not the best strategy to adopt. For this reason we have implemented a new procedure to correct the total sample $(50+15$ galaxies) for velocity dispersion.

We consider the individual fractional index variations $R_{i, j, \sigma}$ and associated line-strength indices $E W_{i, j, \sigma}$ derived for each star at different velocity dispersions. Then for each galactic aperture or gradient the final index correction $R_{i, \sigma}$ is derived 
Table 2. Observing parameters.

\begin{tabular}{ll}
\hline \hline Date of Observations & $10-13$ May 99 \\
Observer & Zeilinger W. \\
Spectrograph & B \& C grating \#25 \\
Detector & ESO CCD \#39, Loral 2K UV flooded \\
Pixel size $(\mu \mathrm{m})$ & 15 \\
Scale along the slit $\left({ }^{\prime \prime} / \mathrm{px}^{-1}\right)$ & 0.82 \\
Slit length (') & 4.5 \\
Slit width (") & 2 \\
Dispersion $\left(\AA \mathrm{mm}^{-1}\right)$ & 187 \\
Spectral resolution $(F W H M$ at $5200 \AA)(\AA)$ & 7.6 \\
Spectral range $(\AA)$ & $3622-9325$ \\
Seeing range $(F W H M)\left({ }^{\prime \prime}\right)$ & $1.2-2$ \\
Standard stars & Feige 56, ltt 1788, ltt 377 \\
\hline
\end{tabular}

Table 3. Journal of galaxy observations.

\begin{tabular}{cccl}
\hline \hline ident. & $\begin{array}{c}\text { Slit PA } \\
{[\text { deg }]}\end{array}$ & $\begin{array}{c}t_{\text {exp }} \\
{[\mathrm{s}]}\end{array}$ & \\
\hline NGC 3607 & 120 & $2 \times 1800$ & \\
NGC 3818 & 103 & $2 \times 1800$ & \\
NGC 4374 & 135 & $2 \times 1800$ & clouds \\
NGC 4696 & 95 & $2 \times 1800$ & clouds \\
NGC 4697 & 70 & $2 \times 1800$ & \\
NGC 5011 & 154 & $2 \times 1800$ & clouds \\
NGC 5044 & 11 & $2 \times 1800$ & low S/N, clouds \\
NGC 5077 & 11 & $2 \times 1800$ & low S/N \\
NGC 5090 & 136 & $2 \times 1800$ & clouds, spectrum includes NGC 5091 spiral \\
NGC 5193 & 71 & $2 \times 1800$ & clouds, spectrum includes a spiral galaxy in the field \\
& & & \\
NGC 5266 & 109 & $2 \times 1800$ & low S/N \\
NGC 5638 & 150 & $2 \times 1800$ & \\
NGC 5812 & 130 & $2 \times 1800$ & clouds \\
NGC 5813 & 145 & $2 \times 1800$ & \\
NGC 5831 & 55 & $2 \times 1800$ & \\
NGC 589 & 30 & $2 \times 1800$ & clouds, strong wind \\
NGC 6758 & 121 & $2 \times 1800$ & \\
NGC 6776 & 15 & $2 \times 1800$ & \\
\hline
\end{tabular}

by interpolating the $R_{i, j, \sigma}$ values at the galactic velocity dispersion $\sigma$ and at the uncorrected index value $E W_{i, \text { old }}$.

For each gradient and aperture the velocity dispersion corrections are computed on the basis of the $\sigma$ values listed in Table 4. The tabulated values characterize the trend of each galaxy velocity dispersion curve. For galaxies having only the central $\left(r_{\mathrm{e}} / 8\right)$ estimate of $\sigma$ we adopt this value also for the correction of the indices at larger radii (the tables of indices uncorrected for velocity dispersion are available on request from the authors).
The new index corrected for the effect of velocity dispersion is computed in the following way:

$E W_{i, \text { new }}=E W_{i, \text { old }} /\left(1+R_{i, \sigma}\right)$.

\subsection{Correction for emission}

The presence of emission lines affects the measure of some line-strength indices. In particular, the $\mathrm{H} \beta$ absorption strength of the underlying stellar population could be contaminated by a 
Table 4. Velocity dispersion values adopted in the correction of the line-strength indices.

\begin{tabular}{lccccccccc}
\hline \hline Ident. & $\begin{array}{c}\sigma_{r_{\mathrm{e}} / 8} \\
{\left[\mathrm{~km} \mathrm{~s}^{-1}\right]}\end{array}$ & $\begin{array}{c}\sigma_{r_{\mathrm{e}} / 4} \\
{\left[\mathrm{~km} \mathrm{~s}^{-1}\right]}\end{array}$ & $\begin{array}{c}\sigma_{r_{\mathrm{e}} / 2} \\
{\left[\mathrm{~km} \mathrm{~s}^{-1}\right]}\end{array}$ & Ref. & Ident. & $\begin{array}{c}\sigma_{r_{\mathrm{e}} / 8} \\
{\left[\mathrm{~km} \mathrm{~s}^{-1}\right]}\end{array}$ & $\begin{array}{c}\sigma_{r_{\mathrm{e}} / 4} \\
{\left[\mathrm{~km} \mathrm{~s}^{-1}\right]}\end{array}$ & $\begin{array}{c}\sigma_{r_{\mathrm{e}} / 2} \\
{\left[\mathrm{~km} \mathrm{~s}^{-1}\right]}\end{array}$ & Ref. \\
\hline NGC 3607 & 220 & 210 & 195 & CMP00 & NGC 5266 & 199 & 140 & & CDB93 \\
NGC 3818 & 191 & 165 & 138 & SP97 & NGC 5638 & 165 & 151 & 132 & Dav83 \\
NGC 4374 & 282 & 283 & 236 & Dav81 & NGC 5812 & 200 & 204 & 172 & B94 \\
NGC 4696 & 254 & 190 & & CDB93 & NGC 5813 & 239 & 220 & 220 & CMP00 \\
NGC 4697 & 174 & 148 & 130 & Pink03 & NGC 5831 & 164 & 143 & 124 & Dav83 \\
NGC 5011 & 249 & 220 & 200 & CDB93 & NGC 5898 & 220 & 183 & 172 & CMP00 \\
NGC 5044 & 239 & 220 & & CMP00 & NGC 6758 & 242 & 210 & 205 & CMP00 \\
NGC 5077 & 260 & 239 & 228 & CMP00 & NGC 6776 & 242 & 214 & 207 & L98 \\
NGC 5090 & 269 & 250 & & CDB93 & & & & & \\
NGC 5193 & 209 & & & & & & & & \\
\hline
\end{tabular}

Notes: the average central value, obtained from the on-line compilation HYPERCAT (http://www-obs.univ-lyon1. fr/hypercat/), is adopted for $\sigma_{r_{\mathrm{e}} / 8}$. At larger radii, $\sigma_{r_{\mathrm{e}} / 4}$ and $\sigma_{r_{\mathrm{e}} / 2}$ are obtained from the references quoted in Cols. 5 and 10. Legend: B94 = Bertin et al. (1994); CDB93 = Carollo et al. (1993); CMP00 = Caon et al. (2000); Dav81 = Davies (1981); Dav83 = Davies et al. (1983); L98 = Longhetti et al. (1998b); Pink03 = Pinkney et al. (2003); SP97 = Simien \& Prugniel (1997a).

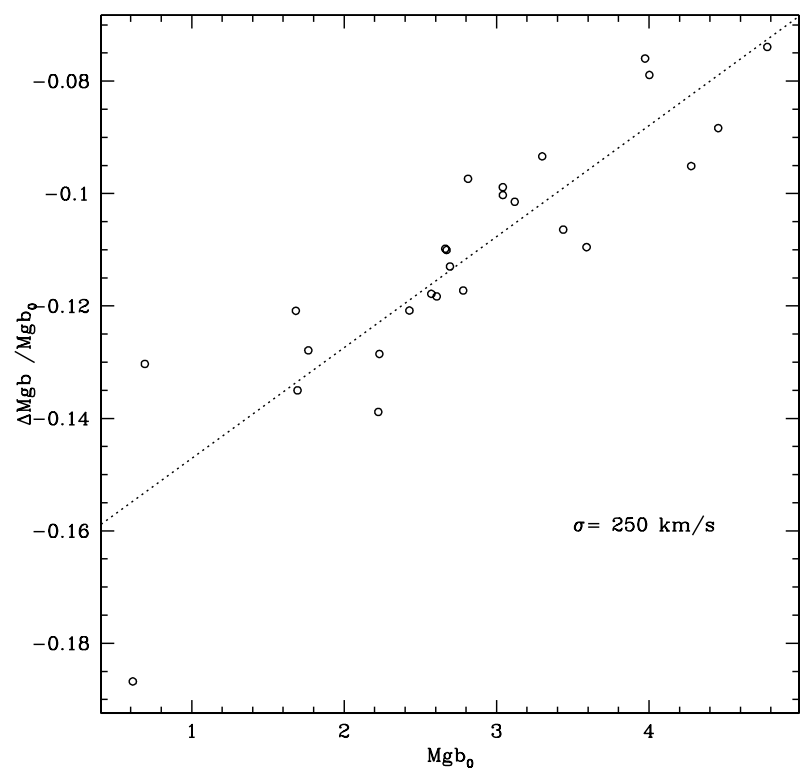

Fig. 3. Fractional variation of the Mgb index for a velocity dispersion $\sigma=250 \mathrm{~km} \mathrm{~s}^{-1}$ as function of $\mathrm{Mgb}_{0}$, the index at zero velocity dispersion. The dotted line is the least squares fit to the data.

significant infilling due to presence of the $\mathrm{H} \beta$ emission component. The adopted correction procedure is detailed in Paper I.

We recall here that the correction of the galaxy line-strength indices is performed through the measure of the $\mathrm{H} \beta$ emission component from the [OIII] emission, according to the relation proposed by G93: $E W\left(\mathrm{H} \beta_{\mathrm{em}}\right) / E W([\mathrm{OIII}] \lambda 5007)=0.7$.

The ([OIII] 25007$)$ emission is derived adopting a template spectrum for the underlying stellar population and measuring in the galaxy spectrum the flux in excess with respect to the template in a band centered around $5007 \AA$. Following Goudfrooij's (1998) suggestion to use the spectrum of an elliptical galaxy to this purpose, we looked among our observed galaxies for those lacking evidence of emission lines or dust in their spectra and images, and selected NGC 1426 as an old population template. Our choice of a galaxy rather than a stellar template, as done instead by G93, is fully motivated in Paper I, to which we refer for details.

Once the template spectrum has been degraded to the velocity dispersion of the galaxy region under exam and normalized to the galaxy continuum, the galaxy and template fluxes are measured within a bandpass (4996.85-5016.85) centered at $5007 \AA$, while the pseduo-continuum is defined by a blue (4885.00-4935.00) and a red (5030.00-5070.00) bandpass (G93). The emitted ([OIII] $\lambda 5007$ ) flux is then derived according to the equation:

$E W_{\mathrm{em}}=\int_{\lambda_{1}}^{\lambda_{2}} \frac{F_{R}-F_{\text {temp }}}{F_{C}} \mathrm{~d} \lambda$

where $F_{\mathrm{R}}, F_{\text {temp }}$ and $F_{\mathrm{C}}$ are the galaxy, the template and the continuum fluxes respectively, while $\lambda_{1}$ and $\lambda_{2}$ define the central band. According to this definition, detected emissions result as negative $E W$ s. Considering the ([OIII $] \lambda 5007)$ emissions detected above $1 \sigma$ (the variance of the spectrum), we derived the $E W$ of the $\mathrm{H} \beta$ emission according to the $\mathrm{G} 93$ relation.

We have compared our derived [OIII] emissions with literature estimates. There are 10 galaxies in common between our complete sample of $(50+15)$ galaxies and the sample of G93, for which Trager et al. (2000) give [OIII] emission (NGC 1453, NGC 3818, NGC 4374, NGC 4552, NGC 4697, NGC 5638, NGC 5812, NGC 5813, NGC 5831, NGC 5846); among the 15 galaxies in common with Denicoló et al. (2005), [OIII] emission is measured for 9 objects (NGC 1052, NGC 1453, NGC 2911, NGC 2974, NGC 3607, NGC 4374, NGC 5363, NGC 5813, NGC 5831). The comparison is shown in the left panel of Fig. 4 for an $r_{\mathrm{e}} / 8$ aperture: open circles and open stars denote respectively the measures of Trager et al. (2000) and Denicoló et al. (2005), while the dotted line marks the one-to-one relation. For the majority of the galaxies our emission estimates are consistent with literature measures within the 

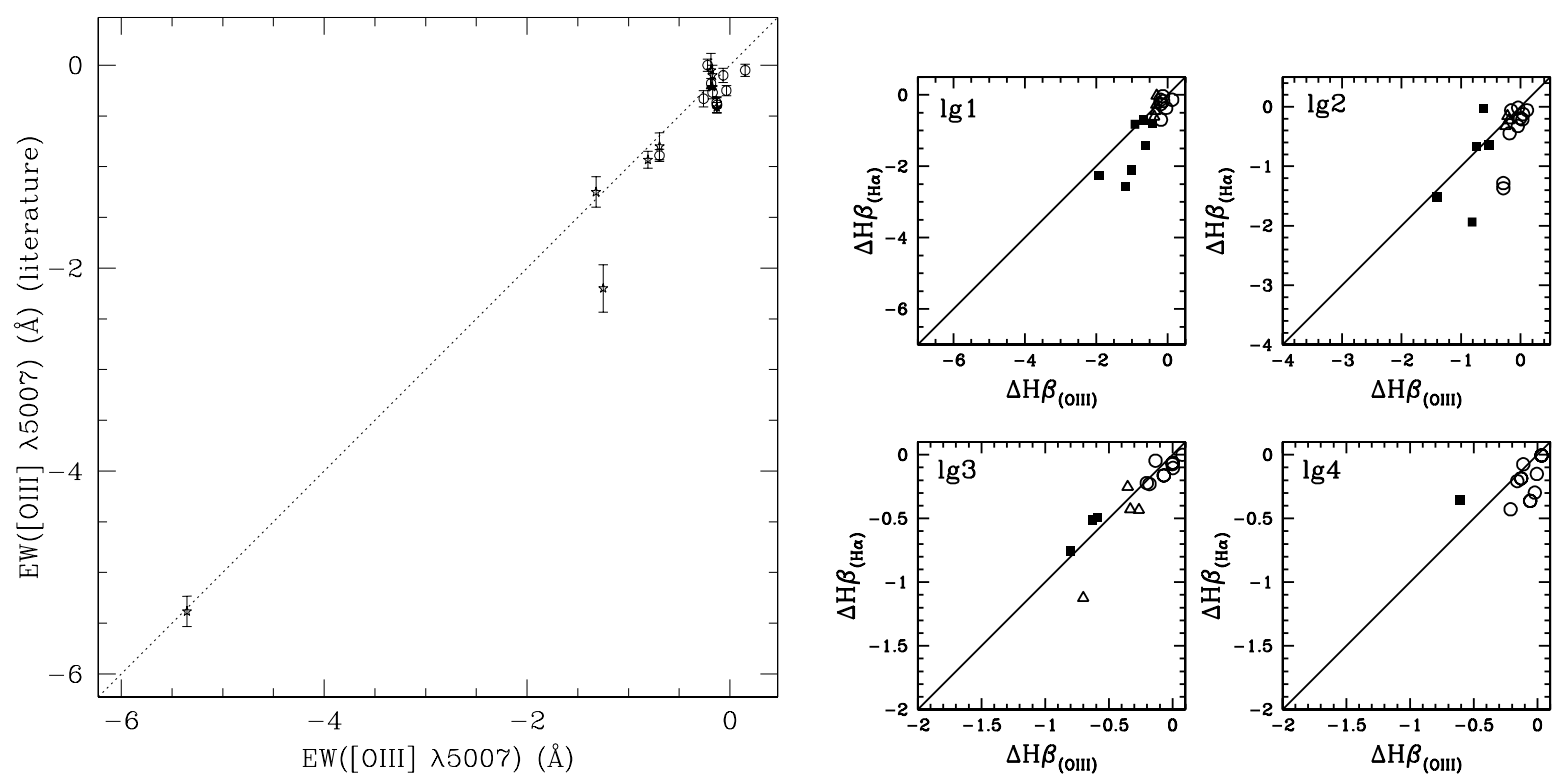

Fig. 4. Left panel: comparison between our ([OIII] $\lambda 5007)$ emission estimates with the measures of Trager et al. (2000) (open circles) and Denicoló et al. (2005) (open stars) within an $r_{\mathrm{e}} / 8$ aperture for the galaxies in common. The dotted line marks the one-to-one relation. Right panel: comparison between the $\mathrm{H} \beta$ emission estimates derived from the ([OIII] $\lambda 5007$ ) and from the $\mathrm{H} \alpha$ emission lines respectively (see Sect. 4.2). The solid line is the one-to-one relation. The comparison is shown in the four regions sampled by the linear gradients (legend: $\lg 1\left(0 \leq r \leq r_{\mathrm{e}} / 16\right.$ ("nuclear")), $\lg 2\left(r_{\mathrm{e}} / 16 \leq r \leq r_{\mathrm{e}} / 8\right), \lg 3\left(r_{\mathrm{e}} / 8 \leq r \leq r_{\mathrm{e}} / 4\right)$ and $\lg 4\left(r_{\mathrm{e}} / 4 \leq r \leq r_{\mathrm{e}} / 2\right)$. Open circles indicate galaxies which $\mathrm{O}[\mathrm{III}]$ emission is detected under $1 \sigma$ level, triangles and full squares between 1 and $2 \sigma$ levels and above $2 \sigma$ level respectively (see text).

errors. The largest deviation from the one-to-one relation is observed for NGC 2911, for which we measure an [OIII] emission of -1.25 , whereas in the literature we find $-2.2 \pm 0.23$.

As done for the original 50 galaxies of the sample of Paper I, we have measured $\mathrm{H} \alpha$ emissions for the 18 galaxies of this paper as well, and have derived the $\mathrm{H} \beta$ emission according to the relation $F_{\mathrm{H} \beta}=1 / 2.86 F_{\mathrm{H} \alpha}$ (see e.g. Osterbrock 1989).

The de-blending of the $\mathrm{H} \alpha$ emission at $\lambda 6563$ from the ([NII] $\lambda 6548,6584$ ) emission lines is performed by fitting each galaxy spectrum (apertures and gradients) with a model resulting from the sum of our old population template galaxy and three Gaussian curves of arbitrary widths and amplitudes (see Fig. 4 of Paper I as an example). Once derived the emitted flux $F_{\mathrm{H} \beta}$ from $F_{\mathrm{H} \alpha}$, we computed the pseudo-continuum in $\mathrm{H} \beta$ according to the bandpass definition of Trager et al. (1998) and used it to transform the emitted flux $F_{\mathrm{H} \beta}$ into $E W$. In the right panel of Fig. 4 we plot the comparison between the $\mathrm{H} \beta$ emission estimates derived from the ([OIII] $\lambda 5007)$ and the $\mathrm{H} \alpha$ lines respectively.

Finally, the corrected $\mathrm{H} \beta_{\text {corr }}$ index is computed from the raw value $\mathrm{H} \beta_{\text {raw }}$ according to the formula $E W\left(\mathrm{H} \beta_{\text {corr }}\right)=$ $E W\left(\mathrm{H} \beta_{\text {raw }}\right)-\mathrm{H} \beta_{\mathrm{em}}$, where $\mathrm{H} \beta_{\mathrm{em}}$ is obtained from the [OIII] emission as previously described. The right panel of Fig. 4 shows that this latter estimate is statistically similar to that obtained from the $\mathrm{H} \alpha$ emission, although the use of the $\mathrm{H} \alpha$ line for emission correction will be widely discussed in a forthcoming paper.

Tables 5 and 6 report the values of the $\mathrm{H} \beta$ correction for the apertures and gradients derived from $\mathrm{O}[\mathrm{III}]$ and $\mathrm{H} \alpha$ (complete tables are given in electronic form).
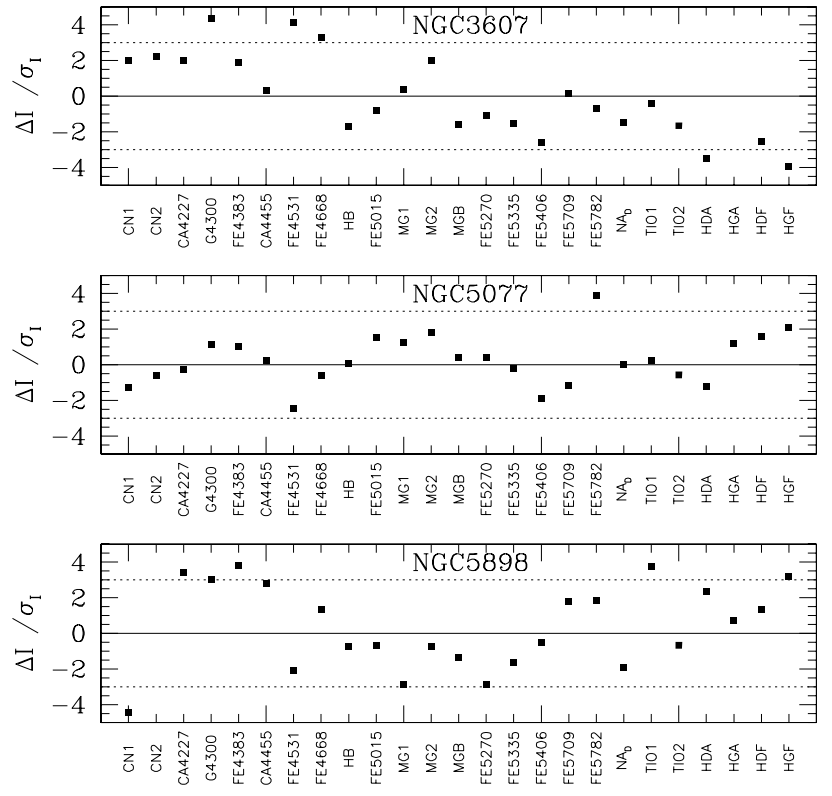

Fig. 5. Comparison of the indices presented Paper I with the measures of this paper for the three galaxies in common (NGC 3607, NGC 5077 and NGC 5898) within an $r_{\mathrm{e}} / 8$ aperture. The index differences are plotted in units of the standard error. The dotted lines delimit the region where $\Delta I / I_{\sigma}<3$.

\subsection{Lick IDS standard stars}

After the indices have been homogenized to the Lick IDS wavelength dependent resolution, corrected for emission and velocity dispersion, they need a final correction to be transformed into the Lick system. The procedure that we adopted is described in Worthey \& Ottaviani (1997) and consists of 

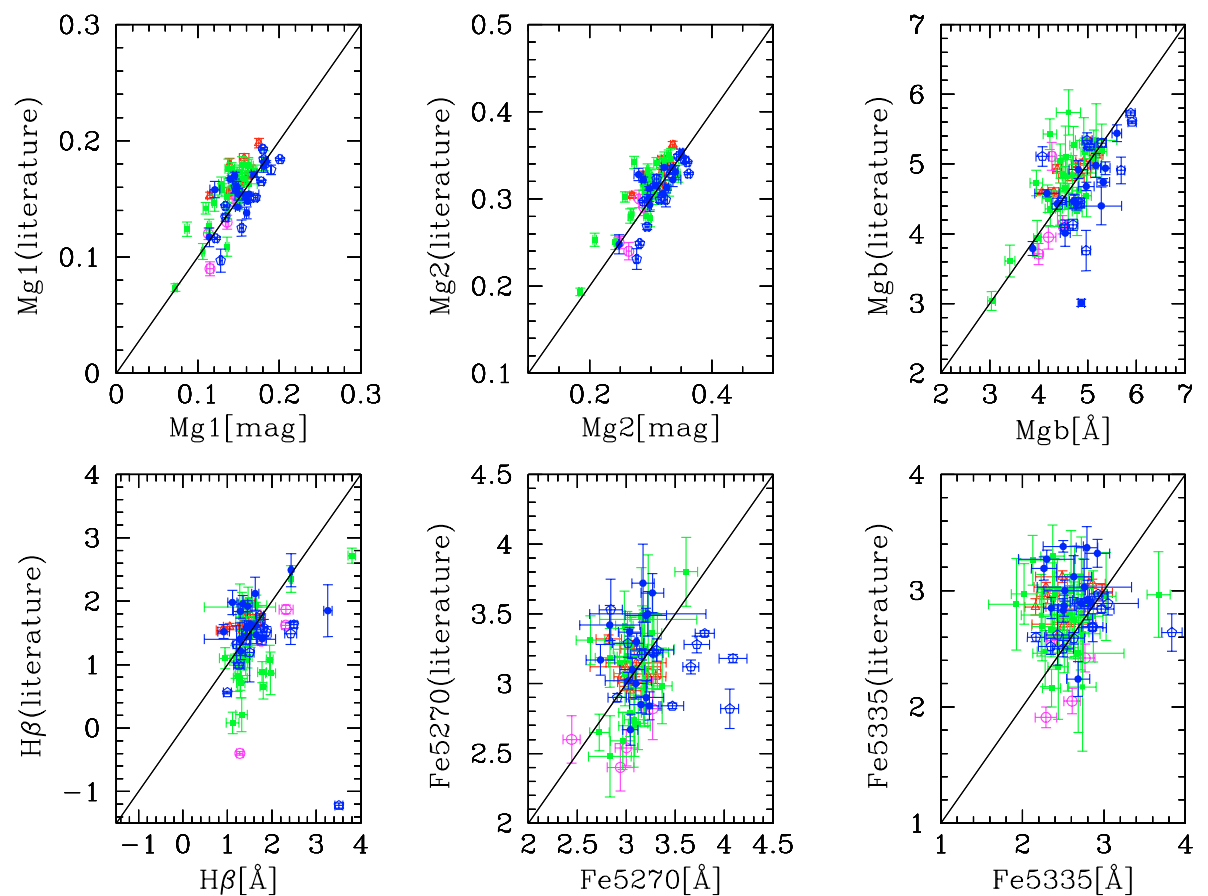

Fig. 6. Comparison of index measurements of González (1993: open triangles), Trager (1998: full squares), Longhetti et al. (1998: open circles), Beuing et al. (2002: open pentagons) and Denicoló et al. (2005: full circles) with our data. Solid lines mark the one-to-one relation. Table 10 summarizes the results of the comparison.

Table 7. $\alpha$ and $\beta$ coeff. for transformation into the Lick system.

\begin{tabular}{|c|c|c|c|c|}
\hline Index & $\alpha$ & $\beta$ & aver. disp. & unit \\
\hline $\mathrm{CN}_{1}$ & 1.059 & 0.023 & 0.025 & $\mathrm{mag}$ \\
\hline $\mathrm{CN}_{2}$ & 1.035 & 0.030 & 0.023 & mag \\
\hline $\mathrm{Ca} 4227$ & 1.317 & 0.408 & 0.396 & $\AA$ \\
\hline G4300 & 1.105 & 0.179 & 0.310 & $\AA$ \\
\hline $\mathrm{Fe} 4383$ & 0.963 & 1.169 & 0.772 & $\AA$ \\
\hline ca4455 & 0.451 & 1.844 & 0.341 & $\AA$ \\
\hline $\mathrm{Fe} 4531$ & 1.289 & -0.299 & 0.437 & $\AA$ \\
\hline $\mathrm{Fe} 4668$ & 0.976 & 0.128 & 0.653 & $\AA$ \\
\hline $\mathrm{H} \beta$ & 1.064 & -0.196 & 0.166 & $\AA$ \\
\hline $\mathrm{Fe} 5015$ & 1.031 & 0.804 & 0.396 & $\AA$ \\
\hline $\mathrm{Mg}_{1}$ & 1.014 & 0.015 & 0.009 & mag \\
\hline $\mathrm{Mg}_{2}$ & 0.998 & 0.020 & 0.012 & mag \\
\hline $\mathrm{Mgb}$ & 1.014 & 0.417 & 0.241 & $\AA$ \\
\hline $\mathrm{Fe} 5270$ & 1.058 & 0.270 & 0.240 & $\AA$ \\
\hline Fe5335 & 0.990 & 0.356 & 0.249 & $\AA$ \\
\hline $\mathrm{Fe} 5406$ & 1.005 & 0.282 & 0.151 & $\AA$ \\
\hline $\mathrm{Fe} 5709$ & 1.321 & -0.270 & 0.174 & $\AA$ \\
\hline $\mathrm{Fe} 5782$ & 1.167 & -0.075 & 0.165 & $\AA$ \\
\hline $\mathrm{NaD}$ & 1.003 & 0.027 & 0.245 & $\AA$ \\
\hline $\mathrm{TiO}_{1}$ & 0.997 & 0.004 & 0.006 & mag \\
\hline $\mathrm{TiO}_{2}$ & 1.003 & -0.001 & 0.008 & mag \\
\hline $\mathrm{H} \delta_{A}$ & 1.136 & -0.622 & 1.087 & $\AA$ \\
\hline $\mathrm{H} \gamma_{A}$ & 0.990 & 0.518 & 0.734 & $\AA$ \\
\hline $\mathrm{H} \delta_{F}$ & 1.059 & -0.036 & 0.503 & $\AA$ \\
\hline $\mathrm{H} \gamma_{F}$ & 1.011 & 0.458 & 0.745 & $\AA$ \\
\hline
\end{tabular}

The table provides the coefficients $\alpha$ and $\beta$ of the transformation into the Lick system $\left(E W_{\text {Lick }}=\beta+\alpha \times E W_{\text {our }}\right)$ derived in Paper I.

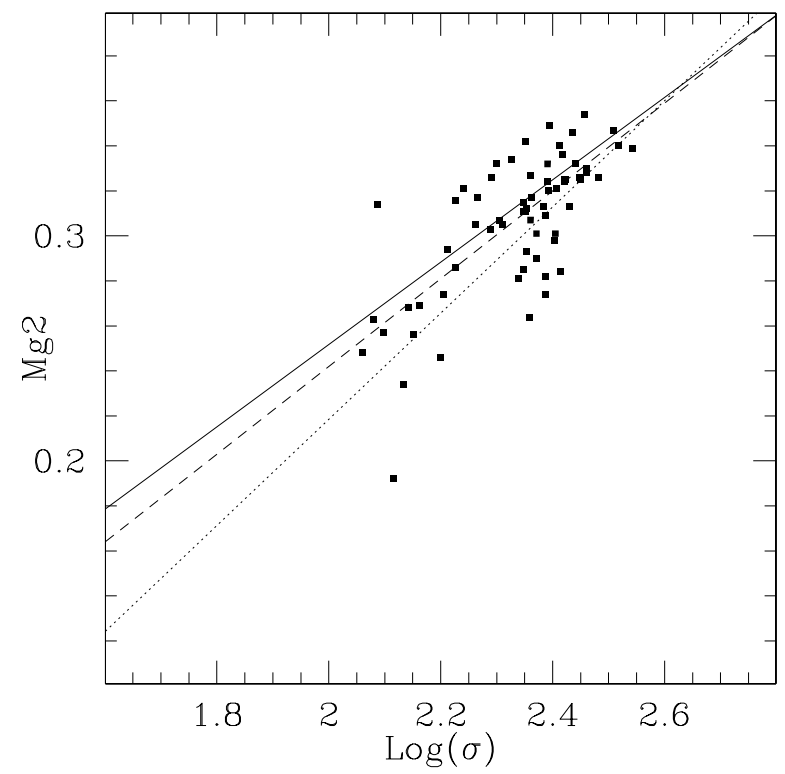

Fig. 7. $\mathrm{Mg}_{2}$ versus $\sigma$ relation. We plot our fully corrected $\mathrm{Mg}_{2}$ linestrength indices within the SLOAN aperture $\left(r=1.5^{\prime \prime}\right)$ versus the corresponding velocity dispersion values. The solid line is the leastsquares fit obtained by Trager et al. (1998). The dotted line represents the least-squares fit obtained by Bernardi et al. (1998) for the field sample of 631 galaxies, while the long-dashed line is our fit (value at $\sigma_{300 \mathrm{~km} \mathrm{~s}^{-1}}=0.335$, slope of relation $\left.=0.195\right)$ to the present data.

observing, contemporary to the galaxies, a sample of standard stars of different spectral types common to the Lick library. In Paper I we derived line strength indices for 17 observed standard stars and compared our measures with the values reported 
by Worthey et al. (1994) in order to derive the transformations into the Lick system. We report in Table 7 the parameters $\alpha$ and $\beta$ of the linear transformation $E W_{\text {Lick }}=\beta+\alpha \times E W_{\text {our }}$ derived in Paper I, where $E W_{\text {our }}$ and $E W_{\text {Lick }}$ are the raw and the Lick indices respectively. The same parameters are used in this paper to perform the transformation into the Lick system. We notice that for the majority of the indices $\alpha$ is very close to 1 and only a zero-point correction is required (see also Puzia et al. 2002), although serious deviations from the one-to-one relation are observed for $\mathrm{Ca} 4227, \mathrm{Ca} 4455$ and $\mathrm{Fe} 4531$.

\subsection{Estimate of index errors}

In order to obtain the errors on each measured index we used the following procedure. Starting from a given extracted spectrum (aperture or gradient at different galactocentric distances), we generated a set of 1000 Monte Carlo random modifications, by adding a wavelength dependent Poissonian fluctuation from the corresponding spectral noise, $\sigma(\lambda)$. Then, for each spectrum, we estimated the moments of the distributions of the 1000 different realizations of its indices.

\section{Results}

For each galaxy of the sample, 25 Lick indices obtained for the 7 apertures and the 4 gradients are provided in electronic form with the format shown in Tables 8 and 9 respectively. We provide also the indices of the original set of Paper I corrected for velocity dispersion according to the new procedure implemented in this paper. The structure of the above tables is the following: each aperture (or gradient) is described by two rows. In the first row: Col. 1 gives the galaxy identification, Col. 2 the number of the aperture, Col. 3 is a flag ( 0 stands for values of indices), Cols. 4 and 5 give the radii delimited by the aperture, from Cols. 6 to 30 individual indices are given. In the second row: Col. 1 gives the galaxy identification, Col. 2 the number of the aperture, Col. 3 is a flag ( 1 stands for error of the indices), Cols. 4 and 5 give the luminosity weighted radius of the aperture and the adopted equivalent radius, from Cols. 6 to 30 the errors of the indices are given. In electronic form are also available, under request to the authors, the tables of the raw indices (before corrections for velocity dispersion, emission and transformation into the Lick system) as well as the fully calibrated spectra (apertures and gradients) in digital form for each galaxy.

In Fig. 5 we compared the indices from Paper I and from this paper for the three galaxies in common (NGC 3607, NGC 5077 and NGC 5898) within an $r_{\mathrm{e}} / 8$ aperture. The figure shows that the two index measures are generally in agreement within $3 \sigma$.

The set of on-line indices in the literature available for a comparison is quite heterogeneous since indices are measured within different apertures. There are ten galaxies in common between the total sample and the G93 sample, namely NGC 1453, NGC 4552, NGC 5846, NGC 3818, NGC 4374, NGC 4697, NGC 5638, NGC 5812, NGC 5813 and NGC 5831. Four galaxies belong to the Longhetti et al. (1998a) sample (NGC 1553, NGC 6958, NGC 7135 and NGC 6776).
Table 10. Comparison with the literature.

\begin{tabular}{|c|c|c|c|c|}
\hline index & $N_{\mathrm{gal}}$ & offset & dispersion & units \\
\hline \multicolumn{5}{|c|}{ G93 + Long98 } \\
\hline $\mathrm{H} \beta$ & 14 & 0.028 & 0.614 & $\AA$ \\
\hline $\operatorname{Mg} 2$ & 14 & -0.012 & 0.021 & mag \\
\hline $\mathrm{Mgb}$ & 14 & -0.136 & 0.375 & $\AA$ \\
\hline $\mathrm{Fe} 5270$ & 14 & 0.069 & 0.295 & $\AA$ \\
\hline $\mathrm{Fe} 5335$ & 14 & -0.169 & 0.446 & $\AA$ \\
\hline Mg1 & 14 & -0.013 & 0.021 & mag \\
\hline \multicolumn{5}{|c|}{ Trager et al. (1998) } \\
\hline $\mathrm{H} \beta$ & 28 & 0.004 & 0.375 & $\AA$ \\
\hline $\operatorname{Mg} 2$ & 28 & -0.016 & 0.026 & mag \\
\hline $\mathrm{Mgb}$ & 28 & -0.203 & 0.447 & $\AA$ \\
\hline $\mathrm{Fe} 5270$ & 29 & -0.094 & 0.506 & $\AA$ \\
\hline $\mathrm{Fe} 5335$ & 29 & -0.241 & 0.504 & $\AA$ \\
\hline $\operatorname{Mg} 1$ & 28 & -0.015 & 0.021 & mag \\
\hline G4300 & 29 & 0.153 & 0.887 & $\AA$ \\
\hline $\mathrm{Ca} 4227$ & 29 & 0.527 & 0.797 & $\AA$ \\
\hline \multicolumn{5}{|c|}{ Beuing et al. (2002) } \\
\hline $\mathrm{H} \beta$ & 12 & -0.043 & 0.273 & $\AA$ \\
\hline $\operatorname{Mg} 2$ & 12 & 0.015 & 0.024 & mag \\
\hline $\mathrm{Mgb}$ & 12 & 0.188 & 0.610 & $\AA$ \\
\hline Fe 5270 & 12 & 0.277 & 0.603 & $\AA$ \\
\hline Fe5335 & 12 & 0.076 & 0.405 & $\AA$ \\
\hline $\operatorname{Mg} 1$ & 12 & 0.008 & 0.018 & mag \\
\hline \multicolumn{5}{|c|}{ Denicoló et al.(2005) } \\
\hline $\mathrm{H} \beta$ & 15 & 0.574 & 1.291 & $\AA$ \\
\hline $\operatorname{Mg} 2$ & 15 & 0.001 & 0.021 & mag \\
\hline $\mathrm{Mgb}$ & 15 & -0.060 & 0.513 & $\AA$ \\
\hline $\mathrm{Fe} 5270$ & 15 & 0.117 & 0.455 & $\AA$ \\
\hline Fe5335 & 15 & -0.052 & 0.333 & $\AA$ \\
\hline $\mathrm{Mg} 1$ & 15 & -0.003 & 0.018 & mag \\
\hline
\end{tabular}

Offsets and dispersions of the residuals between our data and the literature. Dispersions are $1 \sigma$ scatter of the residuals. Beuing et al. (2002) do not compute G4300 and Ca4227 indices. For G4300 and Ca4227 indices Gonzalez (1993) and Longhetti et al. (1998) used a (slightly) different definition than Trager et al. (1998) and consequently comparisons are not reported in the table. The comparison for $\mathrm{H} \beta$ index between our data and those of Trager et al. (1998) and Beuing et al. (2002) are made using uncorrected data.

Twenty nine galaxies are in the sample of Trager et al. (1998) (NGC 128, NGC 777, NGC 1052, NGC 1209, NGC 1380, NGC 1407, NGC 1426, NGC 1453, NGC 1521, NGC 2749, NGC 2962, NGC 2974, NGC 3489, NGC 3607, NGC 3962, NGC 4552, NGC 4636, NGC 5077, NGC 5328, NGC 7332, NGC 7377, NGC 3818, NGC 4374, NGC 4697, NGC 5044, NGC 5638, NGC 5812, NGC 5813, NGC 5831). Twelve galaxies are in the sample published by Beuing et al. (2002), (IC 1459, IC 2006, NGC 1052, NGC 1209, NGC 1407, NGC 1553, NGC 5898, NGC 6868, NGC 6958, NGC 7007, 


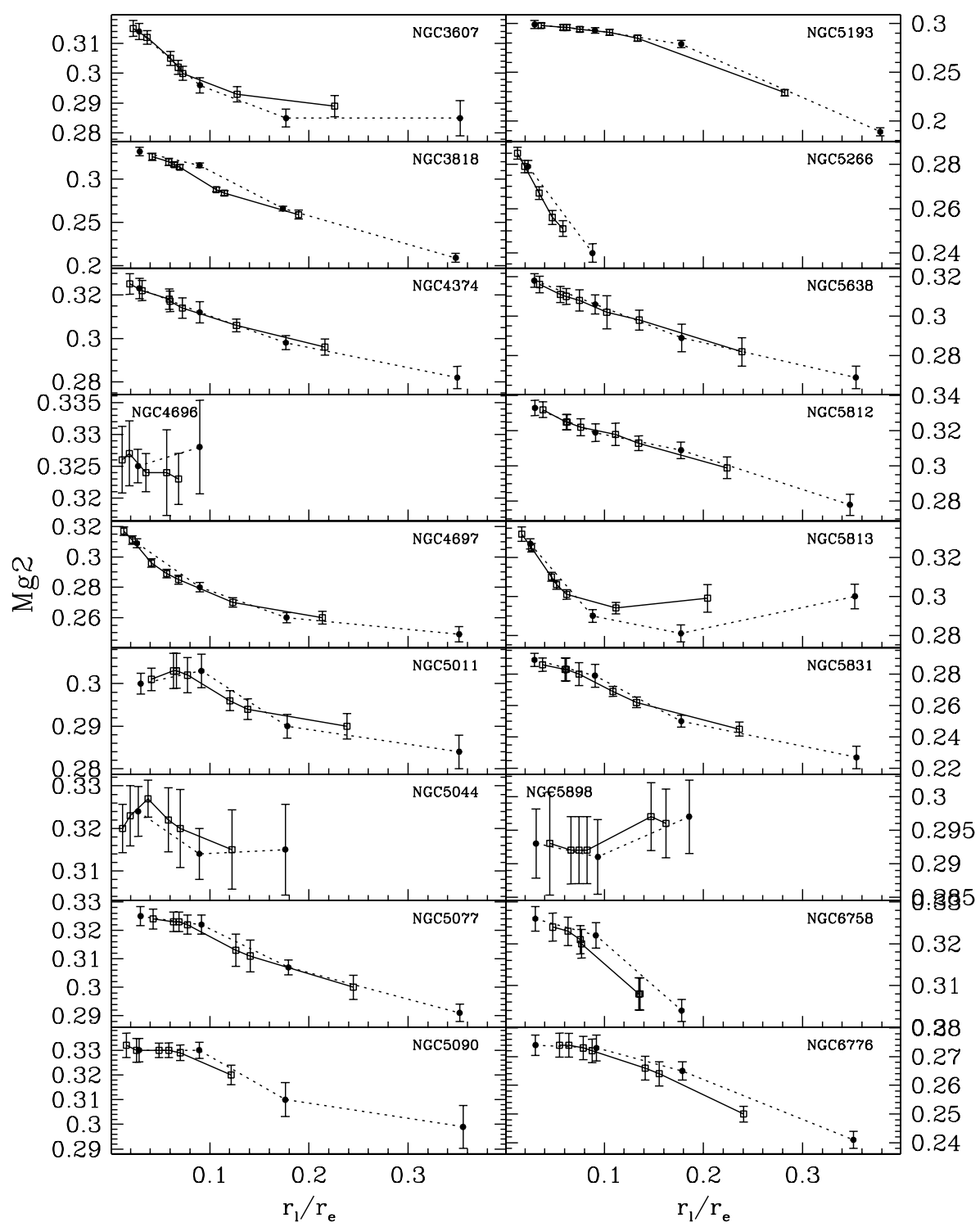

Fig. 8. Fully corrected $\mathrm{Mg}_{2}$ line-strength index as a function of the luminosity weighted radius normalized to the galaxy equivalent radius $R_{\mathrm{e}}$. Apertures are indicated with open squares, while gradients are indicated with full dots.

NGC 7192 and NGC 5812). Finally fifteen galaxies are in common with the sample recently published by Denicoló et al. (2005) (NGC 777, NGC 1052, NGC 1407, NGC 1453, NGC 2911, NGC 2974, NGC 3607, NGC 4374, NGC 5363, NGC 5638, NGC 5812, NGC 5813, NGC 5831, NGC 5846, NGC 7332). The comparison with the literature for the total sample is presented in Fig. 6. In detail: (1) with Longhetti et al. (1998a) the comparison is made with indices computed on the aperture of 2.5" radius; (2) with Gonzalez (1993) on $r_{\mathrm{e}} / 8$ aperture and (3) with Trager et al. (1998) with indices computed within standard apertures; (4) with Beuing et al. (2002) with indices computed on the aperture with radius $r_{\mathrm{e}} / 10$, taking into account that these authors did not correct $\mathrm{H} \beta$ for emission infilling; (5) with Denicoló et al. (2005) on $r_{\mathrm{e}} / 8$ aperture.

Table 10 presents a summary of the comparison with the literature. Both the offset and the dispersion for the various indices in the table are comparable (or better) of those obtained on the same indices by Puzia et al. (2002) in their spectroscopic study of globular clusters. Notice that the discrepancy with Denicoló et al. (2005) regarding the $\mathrm{H} \beta$ index reduces to an offset of 0.090 and a dispersion of 0.404 when the comparison is done with the index values not corrected for emission.

The $\mathrm{Mg}_{2}$ vs. $\sigma$ relation is plotted in Fig. 7 for the total sample of $(50+15)$ galaxies. For a comparison with the SLOAN data, we plot our $\mathrm{Mg}_{2}$ values at the $r=1.5^{\prime \prime}$ versus the corresponding velocity dispersion values. The dotted line is the least-squares fit obtained by Bernardi et al. (1998) on their sample of 631 field early-type galaxies, while the solid line represents the Trager et al. (1998) fit. We recall that the Bernardi et al. (1998) fit is performed on Mg2 index values computed adopting the Lick bandpass definitions, but without transformation into the Lick IDS system. The long-dashed line marks our least-squares fit for the total sample: we observe that our slope is well consistent with that of Bernardi et al. (1998). 


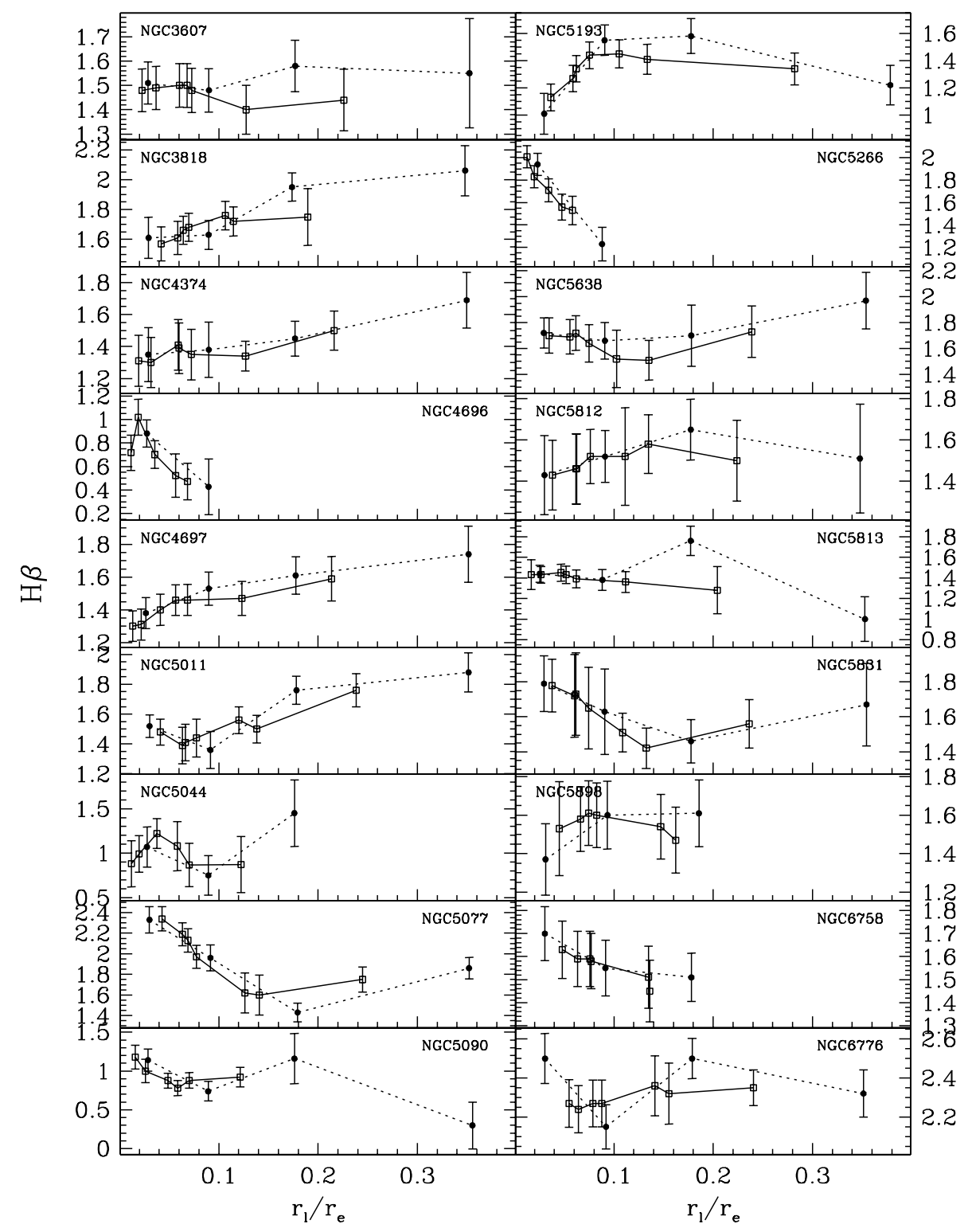

Fig. 9. Fully corrected $\mathrm{H} \beta$ line-strength index as a function of the luminosity weighted radius normalized to the galaxy equivalent radius $R_{\mathrm{e}}$. Apertures are indicated with open squares, while gradients are indicated with full dots.

In Figs. 8-10 we show as examples the trend with radius of the $\mathrm{Mg}_{2}, \mathrm{Fe} 5335$ and $\mathrm{H} \beta$ indices for the 18 galaxies of the sample (apertures are marked with open squares, gradients with dots).

\section{Summary}

This addendum is dedicated, as his Paper I, to the characterization of the underlying stellar population in early-type galaxies with emission lines through the preparation of a data base of their line-strength indices in the Lick IDS system. The indices are measured on the galaxy spectra and then corrected for several effects, more specifically infilling by emission, velocity dispersion and transformation into the Lick IDS system. This paper enlarges the data-set of line-strength indices of 50 earlytype galaxies already presented in Paper I by analyzing 18 additional early-type galaxies (three galaxies, namely NGC 3607,
NGC 5077 and NGC 5898 were already presented in the previous set).

For each object we extracted 7 luminosity weighted apertures (with radii: 1.5", 2.5" $10^{\prime \prime}, r_{\mathrm{e}} / 10, r_{\mathrm{e}} / 8, r_{\mathrm{e}} / 4$ and $r_{\mathrm{e}} / 2$ ) corrected for the galaxy ellipticity and 4 gradients $\left(0 \leq r \leq r_{\mathrm{e}} / 16\right.$, $r_{\mathrm{e}} / 16 \leq r \leq r_{\mathrm{e}} / 8, r_{\mathrm{e}} / 8 \leq r \leq r_{\mathrm{e}} / 4$ and $\left.r_{\mathrm{e}} / 4 \leq r \leq r_{\mathrm{e}} / 2\right)$. For each aperture and gradient we measured 25 line-strength indices: 21 of the original set defined by the Lick IDS "standard" system (Trager et al. 1998) and 4 later introduced by Worthey \& Ottaviani (1997).

Line-strength indices, in particular those used to build the classic $\mathrm{H} \beta-[\mathrm{MgFe}]$ plane, were compared with the literature. A direct comparison was made with González (1993), Longhetti et al. (1998a), Beuing et al. (2002), Trager et al. (1998) and Denicoló et al. (2005), showing the reliability of our measures.

Acknowledgements. R.R. acknowledges the partial support of the ASI (contract I/R/037/01). W.W.Z. acknowledges the support 


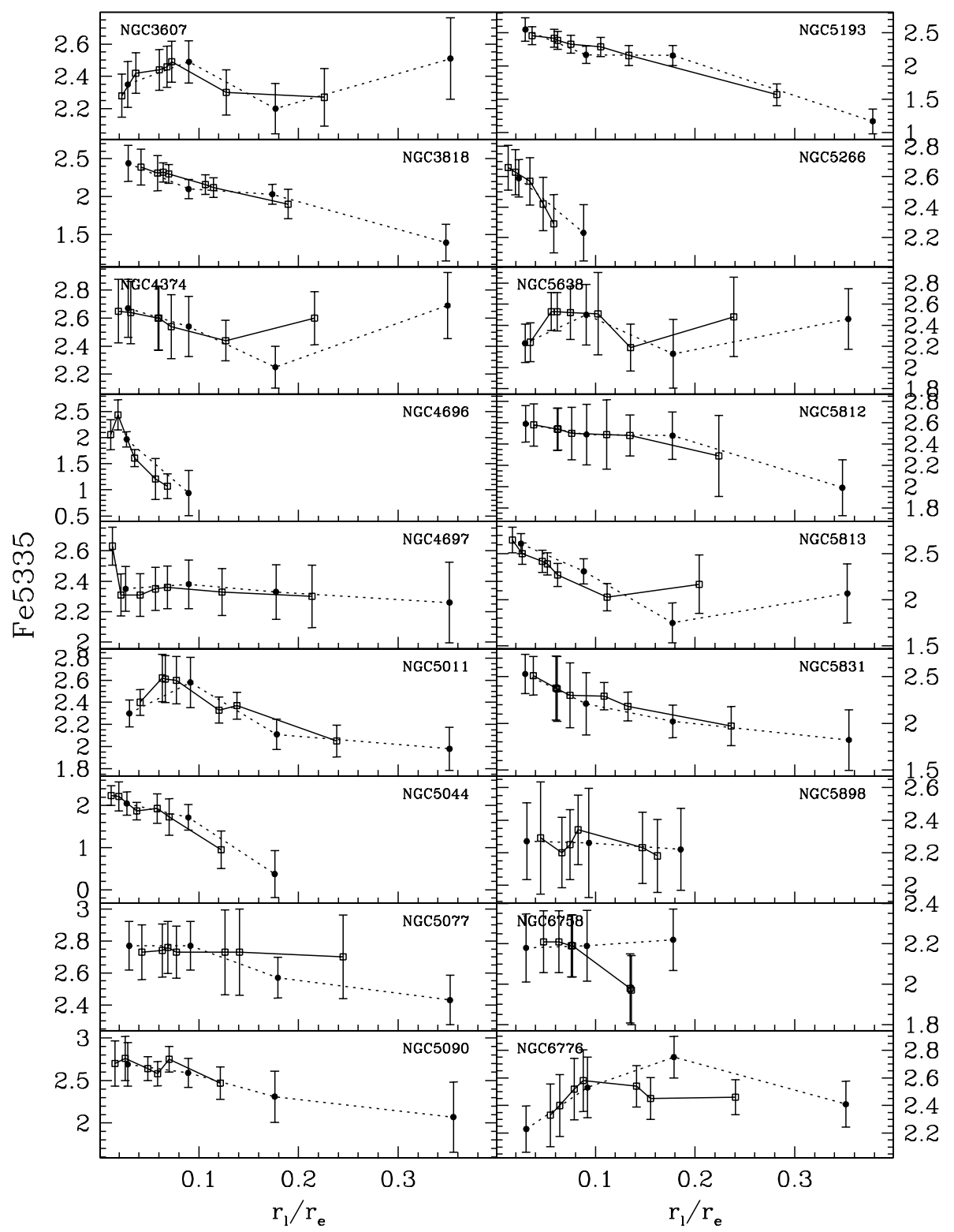

Fig. 10. Fully corrected Fe5335 line-strength index as a function of the luminosity weighted radius normalized to the galaxy equivalent radius $R_{\mathrm{e}}$. Apertures are indicated with open squares, while gradients are indicated with full dots.

of the Austrian Science Fund (project P14783) and of the Bundesministerium für Bildung, Wissenschaft und Kultur. This research has made use of the NASA/IPAC Extragalactic Database (NED) which is operated by the Jet Propulsion Laboratory, California Institute of Technology, under contract with the National Aeronautics and Space Administration.

\section{References}

Allen, S. W., Di Matteo, T., \& Fabian, A. C. 2000, MNRAS, 311, 493 Bernardi, M., Renzini, A., Da Costa, L. N., et al. 1998, ApJ, 508, L43 Bertin, G., Bertola, F., Buson, L., et al. 1994, A\&A, 292, 381

Bertola, F., Capaccioli, M., Galletta, G., \& Rampazzo, R. 1988, A\&A, 192,24

Bettoni, D., Falomo, R., Fasano, G., \& Govoni, F. 2003, A\&A, 399, 869

Beuing, J., Bender, R., Mendes de Oliveira, C., Thomas, D., \& Maraston, C. 2002, A\&A, 395, 431
Bower, G. A., Green, R. F., Quillen, A. C., et al. 2000, ApJ, 534, 189 Buzzoni, A., Gariboldi, G., \& Mantegazza, L. 1992, AJ, 103, 1814 Buzzoni, A., Mantegazza, L., \& Gariboldi, G. 1994, AJ, 107, 513 Caon, N., Capaccioli, M., \& Rampazzo, R. 1990, A\&AS, 86, 429 Caon, N., Macchetto, D., \& Pastoriza, M. 2000, ApJS, 127, 39 Carollo, M., Danziger, I. J., \& Buson, L. M. 1993, MNRAS, 265, 553 Churazov, E., Gilfanov, M., Forman, W., \& Jones, C. 1999, ApJ, 520, 105

Davies, R. L. 1981, MNRAS, 194, 879

Davies, R. L., Efstathiou, G., Fall, S. M., Illingworth, G., \& Schechter, P. L. 1983, ApJ, 266, 41

Denicoló, G., Terlevich, R., Terlevich, E., et al. 2005, MNRAS, 356, 1440

De Vaucouleurs, G., de Vaucouleurs, A., Corwin, H. G. Jr., et al. 1991, Third Reference Catalogue of Bright Galaxies (New York: Springer-Verlag)

Faúndez-Abans, M., \& de Oliveira-Abans, M. 1998, A\&AS, 128, 289

Ferrari, F., Pastoriza, M. G., \& Macchetto, F. D. 2002, A\&A, 389, 355 
Finoguenov, A., \& Jones, C. 2001, ApJ, 547, L107

Gonzalez, J. J. 1993, Ph.D. Thesis, Univ. California, Santa Cruz

Goudfrooij, P. 1994, Ph.D. Thesis, University of Amsterdam, The Netherlands

Goudfrooij, P. 1998, in Star Formation in Early-Type Galaxies, ed. P. Carral, \& J. Cepa, ASP Conf. Ser., 163, 55

Govoni, F., Falomo, R., Fasano, G., \& Scarpa, R. 2000, A\&AS, 143, 369

Kuntschner, H. 2000, MNRAS, 315, 184

Kuntschner, H., Smith, R. J., Colless, M., et al. 2002, MNRAS, 337, 172

Laing, R. A., \& Bridle, A. H. 1987, MNRAS, 228, 557

Lauberts, A., \& Valentijn, E. A. 1989, The Surface Photometry Catalogue of the ESO-Uppsala Galaxies, ESO

Leonardi, A. J., \& Rose, J. A. 1996, AJ, 111, 182

Lewis, K. T., Eracleuos, M., \& Sambruna, R. M. 2003, ApJ, 593, 115

Lloyd, B. D., Jones, P. A., \& Haynes, R. F. 1996, MNRAS, 279, 1187

Longhetti, M., Rampazzo, R., Bressan, A., \& Chiosi, C. 1998a, A\&A, 130,251

Longhetti, M., Rampazzo, R., Bressan, A., \& Chiosi, C. 1998b, A\&A, 130,267

Longhetti, M., Bressan, A., Chiosi, C., \& Rampazzo, R. 1999, A\&A, 345,419

Longhetti, M., Bressan, A., Chiosi, C., \& Rampazzo, R. 2000, A\&A, 353, 917

Macchetto, F., Pastoriza, M., Caon, N., et al. 1996, A\&AS, 120, 463

Malin, D. F., \& Carter, D. 1983, ApJ, 274, 534

Mehlert, D., Thomas, D., Saglia, R. P., Bender, R., \& Wegner, G. 2003, A\&A, 407, 423

Morganti, R., Sadler, E. M., Osterloo, T. A., Pizzella, A., \& Bertola, F. 1997, PASA, 14, 89

Osterbrock, D. 1989, in Astrophysics of Planetary Nebulae and Active Galactic Nuclei (University Science Books)

Peletier, R. F., Davies, R. L., Illingworth, G. D., Davis, L. E., \& Cawson, M. 1990, AJ, 100, 1091

Pierfederici, F., \& Rampazzo, R. 2004, AN, 325, 1
Pinkney, J., Gebhardt, K., Bender, R., et al. 2003, ApJ, 596, 903

Prugniel, Ph., \& Simien, F. 1997, A\&AS, 122, 521

Puzia, T. H., Saglia, R. P., Kissler-Patig, M., et al. 2002, A\&A, 395, 45

Rampazzo, R., Annibali, F., Bressan, A., et al. 2005, A\&A, 433, 497

Reduzzi, L., \& Rampazzo, R. 1995, ApL\&C, 30, 1

Reid, N., Boisson, C., \& Sansom, A. E. 1994, MNRAS, 269, 713

Rest, A., van den Bosch, F., Jaffe, W., et al. 2001, ApJ, 121, 2431

Rickes, M. G., Pastoriza, M. G., \& Bonatto, Ch. 2004, A\&A, 419, 449

Roberts, M. S., Hogg, D. E., Bregman, J. N., Forman, W. R., \& Jones, C. 1991, ApJS, 75, 751

Sage, L., \& Galletta, G. 1993, ApJ, 419, 544

Sandage, A. R., \& Tammann, G. 1987, A Revised Shapley Ames Catalogue of Bright Galaxies, Carnegie, Washington (RSA)

Sansom, A. E., Hibbard, J. E., \& Schweizer, F. 2000, AJ, 120, 1946

Sarazin, C. L., Irwin, J. A., \& Bregman, J. N. 2001, ApJ, 556, 533

Scorza, C., Bender, R., Winkelmann, C., Capaccioli, M., \& Macchetto, D. F. 1998, A\&AS, 131, 265

Simien, F., \& Prugniel, Ph. 1997, A\&AS, 122, 521

Simien, F., \& Prugniel, Ph. 1997a, A\&AS, 126, 15

Sparks, W. B., Carollo, M. C., \& Macchetto, D. F. 1997, ApJ, 486, 253

Thomas, D., Maraston, C., \& Bender, R. 2003, MNRAS, 339, 897

Trager, S. C., Worthey, G., Faber, S. M., Burstein, D., \& Gozalez, J. J. 1998, ApJS, 116, 1

Trager, S. C., Faber, S. M., Worthey, G., \& Gozalez, J. J. 2000, AJ, 119,164

Tully, R. B. 1988, Nearby Galaxy Catalogue (Cambridge University Press)

Varnas, S. R., Bertola, F., Galletta, G., Freeman, K. C., \& Carter, D. 1987, ApJ, 313, 69

Vazdekis, A. 1999, ApJ, 513, 224

Worthey, G. 1992, Ph.D. Thesis, University of California, Santa Cruz

Worthey, G., Faber, S. M., Gonzalez, J. J., \& Burstein, D. 1994, ApJS, 94, 687

Worthey, G., \& Ottaviani, D. L. 1997, ApJS, 111, 377 
F. Annibali et al.: Nearby early-type galaxies with ionized gas. II., Online Material $p 1$

\section{Online Material}




\section{Appendix A: Notes on individual galaxies from the literature}

We report below some studies relevant to the present investigation performed in the recent literature. Attention is paid to the properties of the ionized gas with respect to the bulk of the stellar component and to the cold or hot gas components.

NGC 3818 Scorza et al. (1998) surface photometry suggests that this galaxy belongs to the class of bulge-dominated early-types. In particular, NGC 3818 is representative of the class, having a disk fully embedded in a boxy bulge. The disk profile is exponential all throughout the object. Adopting the values of $V_{\mathrm{M}}=114 \pm 7 \mathrm{~km} \mathrm{~s}^{-1}$ and $\sigma=199 \pm 10$ given by Simien $\&$ Prugniel (1997a) in order to obtain a rough estimate of the position in the $\left(V_{\mathrm{m}} / \sigma, \epsilon\right)$ plane, we may consider the galaxy not far from the region of the oblate galaxies with (nearly) isotropic velocity dispersion. The bulge boxiness suggests a relative peculiarity of this galaxy as indicated by the $\Sigma=1.30$ assigned by Sansom et al. (2000).

NGC 4374, M 84 Caon et al. (1990) provide the $B$-band luminosity profile of this galaxy extending up $\approx 12 r_{\mathrm{e}}$ (at $26 \mu_{B}=$ $26 \mathrm{mag} \operatorname{arcsec}^{-2}$ the galaxy outskirts overlap with those of NGC 4406 (M 86)). The isophote has a nearly constant position angle up to $\approx 80^{\prime \prime}\left(1.6 r_{\mathrm{e}}\right)$ and a strong variation $(\Delta \mathrm{PA} \approx$ $\left.50^{\circ}\right)$ in the outskirts where there are possible contamination by NGC 4406. They notice a complex dust-lane system in the inner 5" which is beautifully visible in the WFPC2 images (see e.g. Bower et al. 2000).

The galaxy stars rotate very slowly $\left(\leq 38 \mathrm{~km} \mathrm{~s}^{-1}\right.$ (Davies et al. 1983 and other rotation velocity determinations in HYPERCAT) which leads to a very low $V_{\mathrm{m}} / \sigma \leq 0.15$, indicating the presence of anisotropy in the velocity dispersion.

WFPC2 high resolution images of NGC 4374 confirm the presence of an extended central ionized gas component (Bower et al. 2000 and reference therein). In the inner $5^{\prime \prime}$ the emission, detected in $\mathrm{H} \alpha+[\mathrm{NII}]$, has three components: a nuclear disk, a ionization cone and outer filaments. The ionization cone is similar to those found in Seyfert galaxies and is also aligned with the radio axes. NGC 4374 is indeed also a Fanaroff-Riley type I (FRI) radio source (Laing \& Bridle 1987).

NGC 4374 is then one of the nearby BH candidate galaxies with a relatively week AGN. The kinematics of the nuclear disk of ionized gas indicates the presence of a central $1.5 \times 10^{9} M_{\odot}$ dark compact object.

Finoguenov \& Jones (2001) analyzed deep Chandra observations of NGC 4374 finding a central AGN, several galactic sources and a diffuse hard emission, where the gas is probably heated by the central AGN. The soft emission instead has the same spatial distribution of the radio structure of the galaxy.

NGC 4696 This galaxy, the dominant member of the Centaurus Cluster, is know to be a radio galaxy PKS1246-41, with a FRI type. Together with the ionized gas, with line ratios typical of a LINER (see Lewis et al. 2003), it possesses neutral gas. This latter is kinematically associated with the compact emission filament system and the dust-lane present in the central 20" of the galaxy (Sparks et al. 1997). Allen et al. (2000) detected hard X-ray emission components in the spectra of NGC 4696. The characteristics of the emission are different from those of Seyfert galaxies, the latter having steeper powerlaw components and higher X-ray luminosities. They argue that the hard X-ray emission is likely to be caused by accretion onto a central, supermassive $\mathrm{BH}$.

The large scale environments of this galaxy is perturbed: ASCA observations show evidence that the main cluster, centred on NGC 4696, is strongly interacting/merging with a subcluster centered on NGC 4709 (see Churazov et al. 1999).

$\underline{\text { NGC } 4697}$ The galaxy is located in the Virgo Cluster southern extension (Tully 1988). The surface photometry of Scorza et al. (1998) shows that the galaxy belongs to the disky family of Es, the faint disk being visible throughout the luminosity profile. In agreement with this finding, no rotation has been measured along the minor axis by Bertola et al. (1988). Goudfrooij et al. (1994) reported a significant detection in both $\mathrm{H} \alpha$ and $[\mathrm{NII}]$ lines with an extension of $\approx 35^{\prime \prime}$.

NGC 4697 is considered a X-ray faint early-type galaxy. Sarazin et al. (2001) observed the galaxy with Chandra, resolving much of the X-ray emission (61\%) into 90 point sources, mostly LMXBs which have lost much of their interstellar gas. The galaxy center hosts a X-ray source which could be an active galactic nucleus (but also one or more LMXBs) radiating at a very small fraction $\left(\leq 4 \times 10^{-8}\right)$ of its Eddington luminosity.

NGC 5044 The galaxy, located in a rich group of galaxies (see e.g. Tully 1988), is rich of dust in the central $10^{\prime \prime}$ with a clumpy distribution, NGC 5044 has been detected by IRAS and by ISO (Ferrari et al. 2002 and reference therein). The gas has a filamentary shape with an extension of about 40" (Macchetto et al. 1996). The gas velocity profile is irregular, with many humps and dips while the inner (within $1 / 3$ of the effective radius) stellar velocity profile is conter-rotating with respect to the outer regions (Caon et al. 2000). The galaxy is, perhaps, a very peculiar object being a possible merger/accretion remnant.

Rickes et al. (2004) studied the ionized gas component in this galaxy suggesting the presence of both a non-thermal ionization source in the central region and an additional ionization source (possibly hot post-AGB stars) in the outer parts.

NGC 5090 The galaxy together with NGC 5091 form the pair RR 242 (Reduzzi \& Rampazzo 1995) which is part of a loose group. Considering a radius of about half a degree around NGC 4105, there are four additional luminous galaxies with comparable redshift: NGC 5082 (separation $5.8^{\prime} 77.5 \mathrm{kpc}$, $\Delta V_{\text {pair }}=421 \mathrm{~km} \mathrm{~s}^{-1}$ ), NGC 5090A (separation 20.3'298.4 kpc, $\Delta V_{\text {pair }}=45 \mathrm{~km} \mathrm{~s}^{-1}$ ), NGC 5090B (separation $13.8^{\prime} 184.1 \mathrm{kpc}, \Delta V_{\text {pair }}=773 \mathrm{~km} \mathrm{~s}^{-1}$ ) and ESO $270 \mathrm{G} 007$ (separation $24.4^{\prime} 326.1 \mathrm{kpc}, \Delta V_{\text {pair }}=275 \mathrm{~km} \mathrm{~s}^{-1}$ ). The group could probably include also ESO 270 G003 (separation 3.5' $46.7 \mathrm{kpc}$ ) whose redshift is still unknown. Considering the above galaxies, the possible loose group has an average recession velocity of $3681 \mathrm{~km} \mathrm{~s}^{-1}$ and a velocity dispersion of $327 \mathrm{~km} \mathrm{~s}^{-1}$. NGC 5090 seems a"bona fide" elliptical without particular signatures of interaction, according to the surface photometry of Govoni et al. (2000). The galaxy hosts an FRI radio source (PKS B1318-434) with two large radio jets and the radio axis perpendicular to the line connecting the nuclei of the pair members (see Llyod et al. 1996 and reference threin). Carollo et al. (1993) have obtained the velocity dispersion and rotation velocity profiles of NGC 5090 
showing a high central velocity dispersion and a low rotation velocity both characteristic of E galaxies. Bettoni et al. (2003), in their study of the $\mathrm{BH}$ mass of low redshift radio galaxies, attributed to NGC 5090 a BH mass of $1.1 \times 10^{9} M_{\odot}$.

NGC 5193 The galaxy forms a physical pair with NGC 5193A, two S0s according to the ESO-LV classification. Reduzzi \& Rampazzo (1995) showed that NGC 5193 is an $\mathrm{E}$, with disky isophotes, while the companion is probably and S0a showing incipient arms. The ongoing interaction of the two galaxies is shown by the warped disk of NGC 5193A. The small disk in NGC 5193 seems aligned with that of the companion, as shown by Faúndez-Abans \& de Oliveira-Abans (1998).

NGC 5266 The galaxy has a prominent dust-lane along its projected minor axis. The kinematics study of Varnas et al. (1987) shows that stars rotate about the optical minor axis while the gas in the dust-lane rotates about the optical major axis, i.e. the kinematic axes of the stars and gas appear othogonal. Varnas et al. (1987) suggest that the underlying galaxy is triaxial.

The ionized gas in NGC 5266 lies in a ring associated with the dust ring Goudfrooij (1994). Sage \& Galletta showed (1993) that the CO has a ring like distribution and that the molecular and ionized gas are co-rotating. Morganti et al. (1997) detected neutral gas up to 8 times the optical effective radius each side of the galaxy. The outer $\mathrm{HI}$ gas extends almost orthogonal to the optical dust-lane: the overall HI kinematics can be modeled by assuming that the gas lies in two orthogonal planes, the plane of the dust-lane, in the central parts, and that perpendicular to this in the outskirts. The large amount of gas, more than $10^{10} M_{\odot}$, and the HI morphology suggest that the galaxy could be the remnant of an old merging episode between two spiral galaxies, since the HI gas appears settled.

NGC 5638 The galaxy forms a physical pair with NGC 5636, an SBa at 2.3' separation. The systemic velocity difference between the two galaxies is $\Delta V=69 \mathrm{~km} \mathrm{~s}^{-1}$. According to the surface photometry of Peletier et al. (1990), NGC 5638 is a rare elliptical with "truly" elliptical isophotes since no significant boxy or disk-like deviations are visible in the $a_{4}$ shape profile. The position angle variation is $\leq 30^{\circ}$ while the $(B-R)$ color is quite stable in the range $\approx 1.6-1.5$ along all the galaxy. The galaxy kinematics along the major axis has been studied by Davies et al. (1983). They found that at about $15^{\prime \prime}$ from the nucleus the galaxy projected rotation velocity is $\approx 90 \mathrm{~km} \mathrm{~s}^{-1}$ with a velocity dispersion of $\approx 120 \mathrm{~km} \mathrm{~s}^{-1}$. The position of the galaxy in the $\left(V_{\mathrm{m}} / \sigma, \epsilon\right)$ plane is consistent with the line traced by oblate galaxies with isotropic velocity dispersion. According to its photometric and kinematical properties, this galaxy should represent a typical "normal" elliptical galaxy.

NGC 5812 Recently, high resolution images have been obtained with WFPC2 (Rest et al. 2001). NGC 5812 has a small dust disk which extends for $0.4^{\prime \prime}$. The luminosity profile has a "power law" shape.

NGC 5812 shows a declining stellar velocity dispersion profile and little rotation $\left(\leq 40 \mathrm{~km} \mathrm{~s}^{-1}\right.$ up to a radius of $25^{\prime \prime}$, i.e. at about $1 r_{\mathrm{e}}$ (Bertin et al. 1994).
NGC 5813 Caon et al. (2000) kinematic study confirms the presence of a decoupled stellar core. The ionized gas has an irregular velocity profile along both the studied directions, suggesting that the gas is still unsettled, a conclusion which is also supported by the filamentary morphology of $\mathrm{H} \alpha+[\mathrm{NII}]$ emission (see also 1996). The WFPC2 image of NGC 5813 suggests the presence of dust in the inner $10^{\prime \prime}$ and a "core-type" luminosity profile (Rest et al. 2001).

NGC 5831 Reid et al. (1994) multicolor photometry indicates that the luminosity profile follows in an excellent way the $r^{1 / 4}$ law at all radii and that the galaxy does not show statistically significant evidence for monotonic changes in color with radius. High resolution images have been obtained with WFPC2. NGC 5831 does not have dust-lane and the luminosity profile has a "power law" shape (Rest et al. 2001) and a significant twisting $\left(\approx 20^{\circ}\right)$ in the inner $20^{\prime \prime}$.

Davies et al. (1983) show that stars in NGC 5831 rotate very slowly along major axis $\left(27 \pm 9 \mathrm{~km} \mathrm{~s}^{-1}\right)$ : the $V / \sigma$ value is 0.18 indicating the presence of anisotropies in the velocity dispersion.

NGC 6758 Caon et al. (2000) measured the gas and star velocity profiles along $\mathrm{PA}=63^{\circ}$ an $\mathrm{PA}=153^{\circ}$. Along $\mathrm{PA}=63^{\circ}$ the gas velocity profile is regular but, after reaching a maximum of about $140 \mathrm{~km} \mathrm{~s}^{-1}$, decreases up to $V=0$. The stars rotate slowly (about $40 \mathrm{~km} \mathrm{~s}^{-1}$ ) and in the opposite sense with respect to the gas. Along $=153^{\circ}$ gas and stars rotate in the same sense. The gas could be then accreted from the outside.

NGC 6776 The galaxy shows a diffuse, asymmetric luminosity distribution and tails. Malin \& Carter (1983) annotated in their catalogue that the fine structure characterizing NGC 6776 seems different from classical shells and it is more reminiscent of a tidal debris forming a loop and tails. The surface photometry made by Pierfederici \& Rampazzo (2004) suggests an E/S0 classification. The isophotes appear strongly twisted in the central region $\left(<20^{\prime \prime}\right)$ and then stabilize around $\mathrm{PA}=20^{\circ}$, suggesting either the presence of a triaxial structure or the presence of two distinct structures. The shape of the profile of the $\mathrm{a}_{4}$ parameter indicates the presence of boxy isophotes, but could also be influenced by the presence of the numerous foreground stars (although they have been properly masked) in addition to intrinsic asymmetries/tails of this galaxy. Pierfederici \& Rampazzo (2004) show that the prominent tail, visible in the E side of the galaxy and extending in the NS direction, has color $(V-R)=0.62 \pm 0.03$ consistent with the average color of the galaxy $\langle V-R\rangle=0.58 \pm 0.04$.

Macchetto et al. (1996) found that the ionized gas component in NGC 6776 has a filamentary structure suggesting that the gas has not yet settled in the galaxy potential. The inner (10" wide) kinematics of NGC 6776 (Longhetti et al. 1998b), shows a regular rotation curve, while the velocity dispersion profile in the same region is asymmetric and shows a plateau $\left(\approx 250 \mathrm{~km} \mathrm{~s}^{-1}\right)$ on the NE side. The study of line strength indices in Longhetti et al. (1999, 2000) suggests that the galaxy had a recent burst $\left(<10^{9}\right.$ years) of star formation. 
F. Annibali et al.: Nearby early-type galaxies with ionized gas. II., Online Material $p 4$

Table 5. $\mathrm{H} \beta$ corrections for apertures.

\begin{tabular}{ccccc}
\hline \hline Galaxy & aperture & $E W \mathrm{O}[\mathrm{III}]$ & Quality & $E W \mathrm{H} \alpha$ \\
\hline NGC 3607 & 0 & -0.342 & 1.000 & -0.651 \\
NGC 3607 & 1 & -0.291 & 1.000 & -0.582 \\
NGC 3607 & 2 & -0.255 & 1.000 & -0.505 \\
NGC 3607 & 3 & -0.246 & 1.000 & -0.519 \\
NGC 3607 & 4 & -0.217 & 1.000 & -0.529 \\
NGC 3607 & 5 & -0.139 & 0.000 & -0.457 \\
NGC 3607 & 6 & -0.104 & 0.000 & -0.271 \\
NGC 3818 & 0 & -0.173 & 0.000 & -0.141 \\
NGC 3818 & 1 & -0.154 & 0.000 & -0.142 \\
NGC 3818 & 2 & -0.182 & 0.000 & -0.154 \\
NGC 3818 & 3 & -0.182 & 0.000 & -0.162 \\
NGC 3818 & 4 & -0.227 & 1.000 & -0.222 \\
NGC 3818 & 5 & -0.178 & 0.000 & -0.235 \\
NGC 3818 & 6 & -0.140 & 0.000 & -0.295 \\
$\ldots \ldots .$. & $\ldots .$. & $\ldots \ldots .$. & $\ldots \ldots$. & $\ldots \ldots$. \\
\hline
\end{tabular}

The table provides the object identification (Col. 1), the number of the aperture (Col. 2), the corresponding radius of which is given in Table 9, and the $E W$ of $\mathrm{H} \beta$ emission as derived from the $\mathrm{O}[\mathrm{III}]$ and $\mathrm{H} \alpha$ emissions (Cols. 3 and 5 respectively). Column 4 gives the quality of the measure, obtained as the ratio between the estimated emission $\left(F_{\text {gal }}-F_{\text {templ }}\right)$ and the variance of the spectrum in the $\mathrm{O}[\mathrm{III}]$ wavelength region, $\sigma_{\lambda}$. When the emission is lower than the variance, the quality is set to 0 . When the emission is between 1 and $2 \sigma_{\lambda}$ or larger than $2 \sigma_{\lambda}$, the quality is set to 1 and 2 respectively. The entire table is given is electronic form.
Table 6. $\mathrm{H} \beta$ corrections for gradients.

\begin{tabular}{ccccc}
\hline \hline Galaxy & aperture & $E W \mathrm{O}[\mathrm{III}]$ & Quality & $E W \mathrm{H} \alpha$ \\
\hline NGC 3607 & 0 & -0.369 & 1.000 & -0.608 \\
NGC 3607 & 1 & -0.183 & 0.000 & -0.445 \\
NGC 3607 & 2 & -0.263 & 1.000 & -0.434 \\
NGC 3607 & 3 & 0.117 & 0.000 & -0.129 \\
NGC 3818 & 0 & -0.1947 & 0.000 & -0.135 \\
NGC 3818 & 1 & -0.220 & 1.000 & -0.153 \\
NGC 3818 & 2 & -0.352 & 1.000 & -0.251 \\
NGC 3818 & 3 & -0.211 & 0.000 & -0.428 \\
$\ldots$. & $\ldots$ & $\ldots .$. & $\ldots .$. & $\ldots .$. \\
\hline
\end{tabular}

The table provides the object identification (Col. 1), the number of the gradient (column 2) $\left(0=0 \leq r \leq r_{\mathrm{e}} / 16,1=r_{\mathrm{e}} / 16 \leq r \leq r_{\mathrm{e}} / 8\right.$, $2=r_{\mathrm{e}} / 8 \leq r \leq r_{\mathrm{e}} / 4$ and $\left.3=r_{\mathrm{e}} / 4 \leq r \leq r_{\mathrm{e}} / 2\right)$, and the $E W$ of $\mathrm{H} \beta$ emission as derived from the $\mathrm{O}[\mathrm{III}]$ and $\mathrm{H} \alpha$ emissions (Cols. 3 and 5 respectively). Column 4 gives the quality of the measure, obtained as the ratio between the estimated emission $\left(F_{\text {gal }}-F_{\text {templ }}\right)$ and the variance of the spectrum in the $\mathrm{O}[\mathrm{III}]$ wavelength region, $\sigma_{\lambda}$. When the emission is lower than the variance, the quality is set to 0 . When the emission is between 1 and $2 \sigma_{\lambda}$ or larger than $2 \sigma_{\lambda}$, the quality is set to 1 and 2 respectively. The entire table is given in electronic form. 
F. Annibali et al.: Nearby early-type galaxies with ionized gas. II., Online Material p 5

Table 8. Fully corrected line-strength indices for the apertures.

\begin{tabular}{|c|c|c|c|c|c|c|c|c|c|c|c|c|c|c|c|}
\hline $\begin{array}{l}\text { galaxy } \\
\text { galaxy }\end{array}$ & $\begin{array}{l}i z \\
i z\end{array}$ & $\begin{array}{l}\text { ie } \\
\text { ie }\end{array}$ & $\begin{array}{l}r_{1} \\
r_{l} \\
\end{array}$ & $\begin{array}{l}r_{2} \\
r_{\mathrm{e}} \\
\end{array}$ & $\begin{array}{l}\mathrm{CN}_{1} \\
\mathrm{eCN}_{1}\end{array}$ & $\begin{array}{l}\mathrm{CN}_{2} \\
\mathrm{eCN}_{2} \\
\end{array}$ & $\begin{array}{c}\mathrm{Ca} 4227 \\
\mathrm{eCa} 4227\end{array}$ & $\begin{array}{c}\text { G4300 } \\
\text { eG4300 }\end{array}$ & $\begin{array}{c}\mathrm{Fe} 4383 \\
\mathrm{eFe} 4383\end{array}$ & $\begin{array}{c}\mathrm{Ca} 4455 \\
\mathrm{eCa} 4455\end{array}$ & $\begin{array}{c}\mathrm{Fe} 4531 \\
\mathrm{eFe} 4531\end{array}$ & $\begin{array}{c}\mathrm{Fe} 4668 \\
\mathrm{eFe} 4668\end{array}$ & $\begin{array}{c}\mathrm{H} \beta \\
\mathrm{eH} \beta\end{array}$ & $\begin{array}{c}\text { Fe5015 } \\
\text { eFe5015 }\end{array}$ & $\begin{array}{l}\text { other } \\
\text { indices }\end{array}$ \\
\hline NGC 3607 & 0 & 0 & 0.000 & 0.035 & 0.146 & 0.181 & 1.630 & 5.940 & 6.040 & 2.420 & 3.610 & 8.580 & 1.480 & 5.720 & $\ldots$ \\
\hline NGC 3607 & 0 & 1 & 0.023 & 43.400 & 0.004 & 0.005 & 0.082 & 0.139 & 0.165 & 0.085 & 0.135 & 0.211 & 0.088 & 0.212 & $\ldots$ \\
\hline NGC 3607 & 1 & 0 & 0.000 & 0.058 & 0.139 & 0.172 & 1.570 & 5.900 & 5.870 & 2.320 & 3.810 & 8.260 & 1.490 & 5.680 & $\ldots$ \\
\hline NGC 3607 & 1 & 1 & 0.037 & 43.400 & 0.003 & 0.003 & 0.055 & 0.093 & 0.152 & 0.079 & 0.138 & 0.225 & 0.089 & 0.231 & $\ldots$ \\
\hline NGC 3607 & 2 & 0 & 0.000 & 0.100 & 0.124 & 0.153 & 1.600 & 5.890 & 5.830 & 2.310 & 3.900 & 7.920 & 1.500 & 5.730 & $\ldots$ \\
\hline NGC 3607 & 2 & 1 & 0.060 & 43.400 & 0.003 & 0.003 & 0.055 & 0.095 & 0.154 & 0.079 & 0.138 & 0.228 & 0.090 & 0.233 & $\ldots$ \\
\hline NGC 3607 & 3 & 0 & 0.000 & 0.115 & 0.119 & 0.150 & 1.580 & 5.830 & 5.800 & 2.310 & 3.860 & 7.750 & 1.500 & 5.760 & $\ldots$ \\
\hline NGC 3607 & 3 & 1 & 0.068 & 43.400 & 0.003 & 0.003 & 0.056 & 0.096 & 0.155 & 0.080 & 0.139 & 0.230 & 0.090 & 0.235 & $\ldots$ \\
\hline NGC 3607 & 4 & 0 & 0.000 & 0.125 & 0.116 & 0.148 & 1.560 & 5.820 & 5.810 & 2.320 & 3.860 & 7.670 & 1.480 & 5.740 & $\ldots$ \\
\hline NGC 3607 & 4 & 1 & 0.073 & 43.400 & 0.003 & 0.003 & 0.056 & 0.097 & 0.155 & 0.080 & 0.139 & 0.231 & 0.091 & 0.236 & ... \\
\hline NGC 3607 & 5 & 0 & 0.000 & 0.250 & 0.097 & 0.129 & 1.500 & 5.770 & 5.680 & 2.330 & 3.710 & 6.970 & 1.400 & 5.600 & $\ldots$ \\
\hline NGC 3607 & 5 & 1 & 0.127 & 43.400 & 0.003 & 0.004 & 0.062 & 0.106 & 0.170 & 0.087 & 0.153 & 0.257 & 0.100 & 0.259 & $\ldots$ \\
\hline NGC 3607 & 6 & 0 & 0.000 & 0.500 & 0.091 & 0.120 & 1.640 & 5.750 & 5.790 & 2.380 & 3.560 & 6.450 & 1.440 & 5.570 & ... \\
\hline NGC 3607 & 6 & 1 & 0.226 & 43.400 & 0.006 & 0.007 & 0.106 & 0.170 & 0.214 & 0.118 & 0.198 & 0.287 & 0.127 & 0.272 & $\ldots$ \\
\hline NGC 3818 & 0 & 0 & 0.000 & 0.068 & 0.143 & 0.183 & 1.650 & 6.170 & 5.800 & 2.320 & 3.620 & 7.400 & 1.570 & 5.800 & $\ldots$ \\
\hline NGC 3818 & 0 & 1 & 0.042 & 22.200 & 0.004 & 0.005 & 0.074 & 0.127 & 0.203 & 0.113 & 0.189 & 0.268 & 0.114 & 0.289 & ... \\
\hline NGC 3818 & 1 & 0 & 0.000 & 0.100 & 0.130 & 0.162 & 1.660 & 6.090 & 5.760 & 2.300 & 3.690 & 7.100 & 1.610 & 5.800 & ... \\
\hline NGC 3818 & 1 & 1 & 0.059 & 22.200 & 0.004 & 0.005 & 0.072 & 0.125 & 0.199 & 0.112 & 0.186 & 0.264 & 0.111 & 0.281 & ... \\
\hline NGC 3818 & 2 & 0 & 0.000 & 0.113 & 0.128 & 0.158 & 1.650 & 6.050 & 5.730 & 2.300 & 3.670 & 6.970 & 1.660 & 5.830 & $\ldots$ \\
\hline NGC 3818 & 2 & 1 & 0.064 & 22.200 & 0.003 & 0.003 & 0.058 & 0.107 & 0.164 & 0.074 & 0.134 & 0.217 & 0.094 & 0.185 & ... \\
\hline NGC 3818 & 3 & 0 & 0.000 & 0.125 & 0.125 & 0.156 & 1.690 & 6.030 & 5.700 & 2.290 & 3.630 & 6.850 & 1.680 & 5.810 & ... \\
\hline NGC 3818 & 3 & 1 & 0.070 & 22.200 & 0.003 & 0.004 & 0.058 & 0.106 & 0.164 & 0.074 & 0.134 & 0.217 & 0.094 & 0.186 & $\ldots$ \\
\hline NGC 3818 & 4 & 0 & 0.000 & 0.225 & 0.100 & 0.130 & 1.570 & 5.960 & 5.320 & 2.270 & 3.390 & 5.990 & 1.760 & 5.560 & $\ldots$ \\
\hline NGC 3818 & 4 & 1 & 0.107 & 22.200 & 0.003 & 0.004 & 0.060 & 0.109 & 0.168 & 0.076 & 0.137 & 0.224 & 0.096 & 0.190 & $\ldots$ \\
\hline NGC 3818 & 5 & 0 & 0.000 & 0.250 & 0.095 & 0.125 & 1.540 & 5.940 & 5.240 & 2.260 & 3.370 & 5.820 & 1.720 & 5.550 & $\ldots$ \\
\hline NGC 3818 & 5 & 1 & 0.115 & 22.200 & 0.003 & 0.004 & 0.061 & 0.110 & 0.171 & 0.077 & 0.139 & 0.228 & 0.097 & 0.194 & $\ldots$ \\
\hline NGC 3818 & 6 & 0 & 0.000 & 0.500 & 0.077 & 0.103 & 1.220 & 5.610 & 4.530 & 2.100 & 3.110 & 5.460 & 1.750 & 5.440 & $\ldots$ \\
\hline NGC 3818 & 6 & 1 & 0.190 & 22.200 & 0.007 & 0.008 & 0.133 & 0.230 & 0.412 & 0.190 & 0.335 & 0.549 & 0.190 & 0.470 & $\ldots$ \\
\hline$\ldots$ & $\ldots$ & $\ldots$ & $\ldots$ & $\ldots$ & $\ldots$ & $\ldots$ & $\ldots$ & $\ldots$ & $\ldots$ & $\ldots$ & $\ldots$ & $\ldots$ & $\ldots$ & $\ldots$ & $\ldots$ \\
\hline
\end{tabular}

First row: galaxy identification (Col. 1), number of the aperture (Col. 2), flag indicating that index values are given (Col. 3), radii delimited by the aperture (Cols. 4 and 5), indices for the apertures (Cols. 6 through 30). Second row: galaxy identification (Col. 1), number of the aperture (Col. 2), flag indicating that error values are given (Col. 3), luminosity weighted radius of the aperture (Col. 4), adopted effective radius (Col. 5), errors on the indices (Cols. 6 through 30). The complete Table 8 is given in electronic form. 
F. Annibali et al.: Nearby early-type galaxies with ionized gas. II., Online Material $p 6$

Table 9. Fully corrected line-strength indices for the gradients.

\begin{tabular}{|c|c|c|c|c|c|c|c|c|c|c|c|c|c|c|c|}
\hline $\begin{array}{l}\text { galaxy } \\
\text { galaxy }\end{array}$ & $\begin{array}{l}i z \\
i z\end{array}$ & $\begin{array}{l}\text { ie } \\
\text { ie }\end{array}$ & $\begin{array}{l}r_{1} \\
r_{l}\end{array}$ & $\begin{array}{l}r_{2} \\
r_{\mathrm{e}} \\
\end{array}$ & $\begin{array}{l}\mathrm{CN}_{1} \\
\mathrm{eCN}_{1} \\
\end{array}$ & $\begin{array}{l}\mathrm{CN}_{2} \\
\mathrm{eCN}_{2} \\
\end{array}$ & $\begin{array}{c}\mathrm{Ca} 4227 \\
\mathrm{eCa} 4227\end{array}$ & $\begin{array}{c}\text { G4300 } \\
\text { eG4300 }\end{array}$ & $\begin{array}{c}\mathrm{Fe} 4383 \\
\mathrm{eFe} 4383\end{array}$ & $\begin{array}{c}\mathrm{Ca} 4455 \\
\mathrm{eCa} 4455\end{array}$ & $\begin{array}{c}\mathrm{Fe} 4531 \\
\mathrm{eFe} 4531\end{array}$ & $\begin{array}{c}\mathrm{Fe} 4668 \\
\mathrm{eFe} 4668\end{array}$ & $\begin{array}{c}\mathrm{H} \beta \\
\mathrm{eH} \beta\end{array}$ & $\begin{array}{c}\text { Fe5015 } \\
\text { eFe5015 }\end{array}$ & $\begin{array}{c}\text { other } \\
\text { indices }\end{array}$ \\
\hline NGC 3607 & 0 & 0 & 0.000 & 0.063 & 0.144 & 0.182 & 1.640 & 5.950 & 6.060 & 2.410 & 3.700 & 8.390 & 1.510 & 5.750 & $\ldots$ \\
\hline NGC 3607 & 0 & 1 & 0.029 & 43.400 & 0.004 & 0.005 & 0.077 & 0.132 & 0.144 & 0.080 & 0.149 & 0.204 & 0.086 & 0.227 & $\ldots$ \\
\hline NGC 3607 & 1 & 0 & 0.063 & 0.125 & 0.106 & 0.135 & 1.570 & 5.800 & 5.780 & 2.310 & 3.940 & 7.450 & 1.480 & 5.760 & $\ldots$ \\
\hline NGC 3607 & 1 & 1 & 0.090 & 43.400 & 0.003 & 0.004 & 0.061 & 0.100 & 0.149 & 0.087 & 0.151 & 0.239 & 0.089 & 0.249 & $\ldots$ \\
\hline NGC 3607 & 2 & 0 & 0.125 & 0.250 & 0.079 & 0.110 & 1.410 & 5.730 & 5.570 & 2.340 & 3.580 & 6.170 & 1.580 & 5.580 & $\ldots$ \\
\hline NGC 3607 & 2 & 1 & 0.177 & 43.400 & 0.004 & 0.004 & 0.072 & 0.118 & 0.177 & 0.101 & 0.178 & 0.287 & 0.106 & 0.294 & $\ldots$ \\
\hline NGC 3607 & 3 & 0 & 0.250 & 0.500 & 0.082 & 0.111 & 1.690 & 5.670 & 5.790 & 2.330 & 3.350 & 6.020 & 1.550 & 5.550 & $\ldots$ \\
\hline NGC 3607 & 3 & 1 & 0.353 & 43.400 & 0.008 & 0.010 & 0.142 & 0.265 & 0.400 & 0.215 & 0.356 & 0.564 & 0.225 & 0.461 & $\ldots$ \\
\hline NGC 3818 & 0 & 0 & 0.000 & 0.063 & 0.158 & 0.194 & 1.670 & 6.120 & 5.860 & 2.350 & 3.580 & 7.630 & 1.610 & 5.870 & $\ldots$ \\
\hline NGC 3818 & 0 & 1 & 0.029 & 22.200 & 0.004 & 0.005 & 0.076 & 0.137 & 0.198 & 0.107 & 0.185 & 0.285 & 0.138 & 0.299 & $\ldots$ \\
\hline NGC 3818 & 1 & 0 & 0.063 & 0.125 & 0.122 & 0.153 & 1.700 & 6.090 & 5.760 & 2.310 & 3.900 & 6.970 & 1.630 & 5.760 & $\ldots$ \\
\hline NGC 3818 & 1 & 1 & 0.090 & 22.200 & 0.003 & 0.004 & 0.066 & 0.111 & 0.171 & 0.087 & 0.145 & 0.245 & 0.096 & 0.250 & $\ldots$ \\
\hline NGC 3818 & 2 & 0 & 0.125 & 0.250 & 0.077 & 0.103 & 1.480 & 5.780 & 4.910 & 2.220 & 2.940 & 5.140 & 1.950 & 5.450 & ... \\
\hline NGC 3818 & 2 & 1 & 0.174 & 22.200 & 0.003 & 0.004 & 0.069 & 0.116 & 0.179 & 0.090 & 0.148 & 0.242 & 0.094 & 0.237 & ... \\
\hline NGC 3818 & 3 & 0 & 0.250 & 0.500 & 0.037 & 0.065 & 0.904 & 5.380 & 3.500 & 1.990 & 3.080 & 4.040 & 2.060 & 5.480 & ... \\
\hline NGC 3818 & 3 & 1 & 0.348 & 22.200 & 0.006 & 0.007 & 0.123 & 0.199 & 0.326 & 0.165 & 0.268 & 0.458 & 0.168 & 0.422 & $\ldots$ \\
\hline
\end{tabular}

First row: galaxy identification (Col. 1), number of the gradient (Col. 2), flag indicating that index values are given (Col. 3), radii delimited by the gradient (Cols. 4 and 5), indices for the gradients (Cols. 6 through 30). Second row: galaxy identification (Col. 1), number of the gradient (Col. 2), flag indicating that error values are given (Col. 3), luminosity weighted radius of the gradient (Col. 4), adopted effective radius (Col. 5), errors on the indices (Cols. 6 through 30). The complete Table 9 is given in electronic form. 